\title{
POLITECNICO
}

MILANO 1863

RE.PUBLIC@POLIMI Research Publications at Politecnico di Milano

[Article] An Adjoint Proper Orthogonal Decomposition method for a neutronics reduced order model

Original Citation:

Lorenzi, S., 2018. An Adjoint Proper Orthogonal Decomposition method for a neutronics reduced order model. Annals of Nuclear Energy, 114, 245 - 258. ISSN:0306-4549

Availability:

This version is available at http://hdl.handle.net/11311/1048463 since March 2018

Publisher:

Elsevier

Published version - when citing, please refer to the published version:

DOI 10.1016/j.anucene.2017.12.029

\section{Publisher copyright}

Elsevier

(C) 2018. This manuscript version is made available under the CC-BY-NC-ND 4.0 license http://creativecommons.org/licenses/by-nc-nd/4.0/

(Article begins on next page) 


\title{
An Adjoint Proper Orthogonal Decomposition method for a neutronics reduced order model
}

\author{
Stefano Lorenzi ${ }^{1, *}$ \\ E-mail: stefano.lorenzi@polimi.it
}

${ }^{1}$ Politecnico di Milano - Department of Energy

via la Masa 34 - 20156, Milano, Italy

\begin{abstract}
This paper deals with the use of Reduced Order Methods for neutronics modelling. This approach is used whether both accuracy and computational efficiency are required. A very popular category of these methods is based on projection approaches which use spatial basis and test functions for the development of the reduced order model. The selection of the spatial basis and test functions used in the projection phase is a crucial issue since it has an impact on the accuracy and the computational cost. In this work, different methods for the creation of the spatial basis and the test functions are analysed. In particular, an Adjoint Proper Orthogonal Decomposition (APOD) method is proposed combining the properties of the Proper Orthogonal Decomposition and the use of the adjoint flux as test function in the neutronics framework. The different methods are applied to create a spatial neutronics model for the ALFRED reactor. The simulation results show that the APOD method gives better results compared to the other methods (Modal Method and standard Proper Orthogonal Decomposition) increasing the accuracy of the reduced order model or minimizing the computational cost.
\end{abstract}

Keywords: Reduced order modelling; Modal Method; Proper Orthogonal Decomposition; Spatial neutronics model; Lead-cooled Fast Reactor

\footnotetext{
* Corresponding author: Politecnico di Milano, via La Masa 34, 20156 Milano, Italy. Tel.: +39 0223996326.

Email address: stefano.lorenzi@ polimi.it (S. Lorenzi).
} 


\section{Introduction}

Different approaches can be found for the neutronics modelling with different degrees of accuracy and related computational effort, e.g., Monte Carlo method, deterministic transport theory, diffusion approximation, Point Kinetics (PK). The selection of the proper approach, according to the specific simulation and analysis needs, is crucial. In this regard, the recent developments in the simulation of nuclear reactors, along with the increased availability of computational resources, do not cancel the importance of having tools that provide useful insights at reasonable computational time. The adoption of Reduced Order Methods (ROMs) (Hesthaven et al., 2016; Rozza et al., 2008) can be suitable for this aim, especially in the areas of process optimization, control or uncertainty quantification (Chinesta et al., 2016; Gunzburger, 2002; Quarteroni et al., 2011). The interest in Reduced Order Methods for the simulation of complex systems in nuclear field is increased in the last years, being applied to Monte Carlo methods (Aufiero et al., 2016; Aufiero and Fratoni, 2017), to the deterministic transport equations (Bang et al., 2015; Buchan et al., 2015), and diffusion problems (Buchan et al., 2013; Gong et al., 2016; Lorenzi et al., 2015; Sartori et al., 2014). The fields of application of ROMs are not limited to the neutronics modelling but they are employed also in sensitivity analysis and uncertainty quantification (Abdel-Khalik et al., 2013; Bang et al., 2012a) and in thermal-hydraulic context (Lorenzi et al., 2017, 2016). Different from the Surrogate Response Surfaces (SRS) approach which are based on data fitting, the methods employed in this work belong to the projection-based family. These Computation Reduction Techniques (CRT) are aimed at reducing the dimension of the algebraic system through the projection onto a small subspace made by global basis functions (Manzoni et al., 2012). As for the neutronics, this paradigm can be applied separating the spatial and time dependence of the neutron flux. The latter can be represented as a linear combination of spatial basis functions calculated from an accurate neutronics modelling weighted by time-dependent coefficients. The dynamic behaviour of the flux is reduced to the study of these time-dependent coefficients, and can be represented by a set of Ordinary Differential Equations (ODEs). This set is obtained multiplying the governing Partial Differential Equations (PDEs) with suitable test functions, as in a Petrov-Galerkin projection. The selection of the spatial basis and test functions is a crucial issue in the development of the reduced order model. In particular, the aim is selecting the optimal pair of spatial basis/test functions that maximizes the accuracy of the model and minimizes the computational cost.

In this work, different approaches in the calculation of both the spatial basis and the test functions are assessed in terms of efficiency (considered as the ratio between accuracy and computational time). As for the spatial basis calculation, the classic option is the Modal Method (MM) that employs as spatial basis the eigenfunctions of the neutron diffusion PDEs calculated in a reference configuration (Stacey, 1969). The Modal Method provides a "general" spatial basis since it is related to the eigenvalue problem of the system, referring to a reference configuration. On the other hand, the natural alternative is to build an ad-hoc spatial basis tailored on specific simulations. This is the case of the Proper Orthogonal Decomposition (POD) with the snapshot technique (Holmes et al., 1996; Sirovich, 1987). In this case, some proper solutions of the neutron diffusion PDEs have to be calculated (i.e., the "snapshots") and the most relevant modes are selected. As for the test functions, the classic option is to use the same functions that constitute the spatial basis as in a Galerkin projection. Nevertheless, the test functions can be different as in a Petrov-Galerkin projection. In this work, an Adjoint Proper Orthogonal Decomposition approach has been developed to combine the 
properties of the Proper Orthogonal Decomposition and the use of the adjoint flux as test function in the neutronics framework.

The different approaches are applied to build a spatial neutronics model of the Advanced Lead Fast Reactor European Demonstrator (ALFRED) (Alemberti et al., 2014). The correct reproduction of the reactivity insertion following a temperature or a Control Rod (CR) change is the main quantity of interest in our case. In this regard, the study of a Lead Fast Reactor represents an interesting case study since in this nuclear system the impact of the coolant density variation may act in different directions (i.e., with a positive or negative local coefficient) according to the core zone involved. This spatial feature cannot be captured with a simplified neutronics modelling (i.e., the Point Kinetics), and a reduced order model can be the desired compromise between accuracy and computational time.

The paper is organized as follows. In Section 2, the ALFRED plant layout and the core configuration are briefly introduced. In Section 3, the modelling approach employed for the spatial neutronics model is presented with the description of the several phases involved in the procedure, with a particular attention to the spatial basis and test functions selection. The simulation results of the ALFRED full core modelling are presented in Section 4, analysing the optimal spatial basis/test functions pair and the performance of the several methods in assessing the reactivity following a temperature change and a CR movement. Finally, some conclusions, perspectives and further developments are given in Section 5.

\section{The Advanced Lead Fast Reactor European Demonstrator}

The reference reactor in this work is the Advanced Lead-cooled Fast Reactor European Demonstrator (ALFRED), developed within the European FP7 LEADER Project. The Project efforts were mainly focused on the resolution of the key issues that emerged in the previous Euratom ELSY Project (Cinotti et al., 2008) to reach a new reference reactor configuration, which was used to design a fully representative scaled-down prototype. The demonstration ALFRED unit is expected to be built at ICN (Institute de Cercetari Nucleare) facility near Pitesti in southern Romania (Alemberti et al., 2013a).

ALFRED is a small-size (300 $\mathrm{MW}_{\text {th }}$ ) pool-type LFR. The current configuration of its primary system (Alemberti et al., 2013b) is depicted in Figure 1. All the major reactor primary system components, including core, primary pumps, and Steam Generators (SGs), are contained within the reactor vessel, being located in a large lead pool inside the reactor tank. The coolant flow coming from the cold pool enters the core and, having passed through the latter, is collected in a volume (hot collector) to be distributed to eight parallel pipes and delivered to as many SGs. After leaving the SGs, the coolant enters the cold pool through the cold leg and returns to the core.

The ALFRED core is composed of wrapped hexagonal Fuel Assemblies (FAs) with pins arranged on a triangular lattice (Figure 2). The 171 FAs are subdivided into two radial zones with different plutonium fractions guaranteeing an effective power flattening, and surrounded by two rows of dummy elements (geometrically identical to the fuel assemblies but not producing thermal power) serving as a reflector. Two different and independent control rod systems have been foreseen, namely, Control Rods (CRs) and Safety Rods (SRs). Power regulation and reactivity swing compensation during the cycle are performed by the former, while the simultaneous use of both is foreseen for scram 
purposes, assuring the required reliability for a safe shutdown (Grasso et al., 2014). In Table 1, the main nominal parameters of ALFRED are presented.

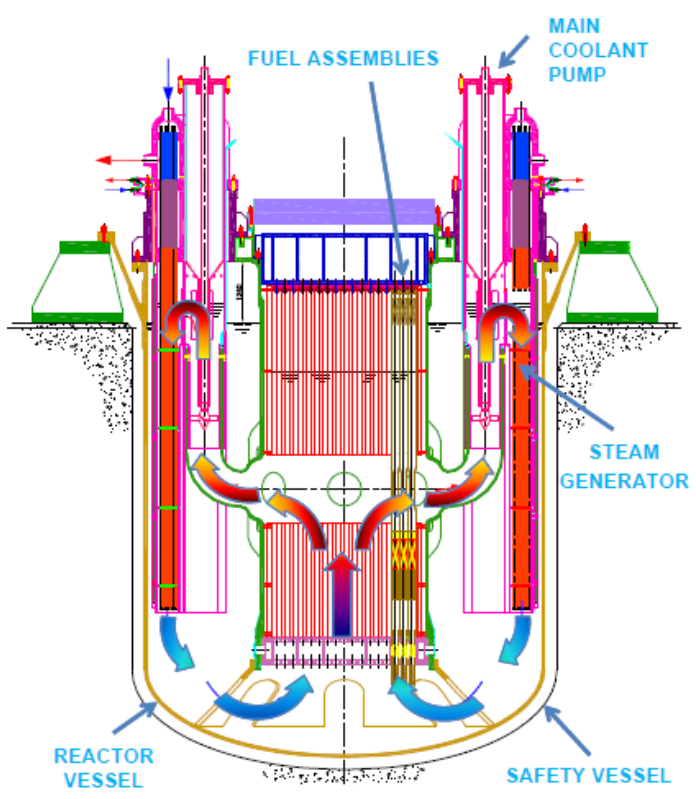

(a)

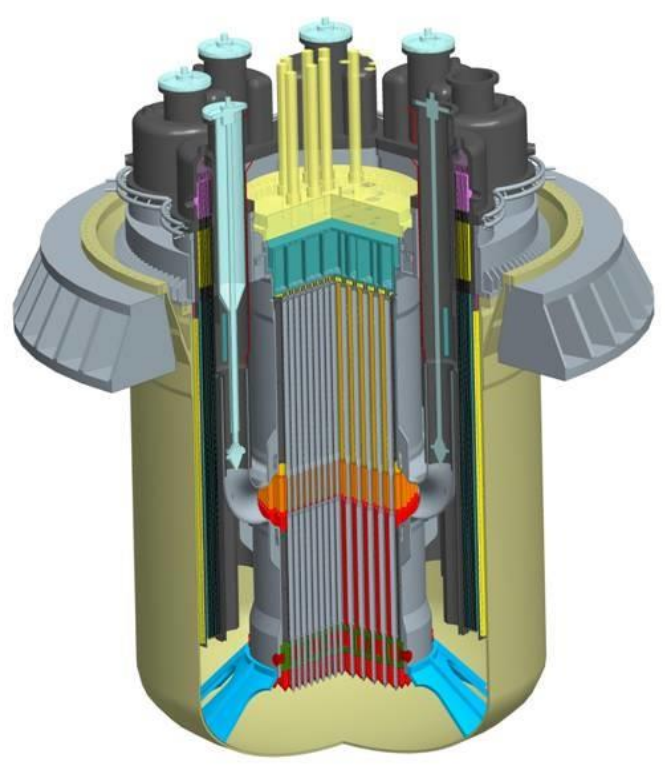

(b)

Figure 1. (a) Reactor vertical section of ALFRED nuclear power plant. (b) 3D representation of the ALFRED reactor (Alemberti et al., 2013b).

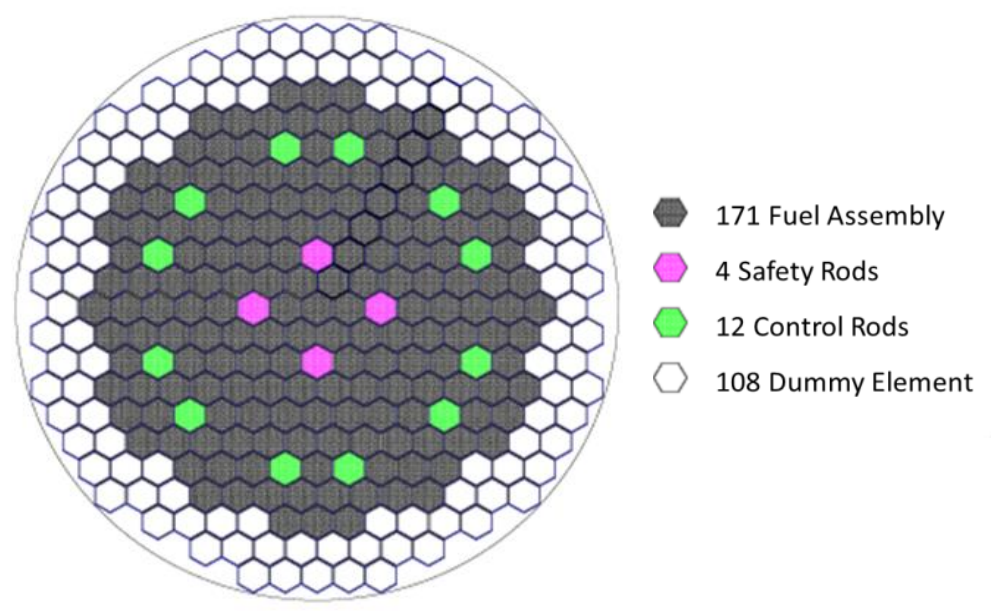

Figure 2. ALFRED core configuration (Grasso et al., 2014). In the reactor, there are 171 fuel assemblies (in grey) surrounded by 108 dummy elements (in white). In the active zone, 4 safety rods (in purple) and 12 control rods (in green) are present.

Table 1. ALFRED core parameters (Grasso et al., 2014).

\begin{tabular}{lcc}
\hline Parameter & Value & Unit \\
\hline Core & & \\
\hline Thermal power & 300 & $\mathrm{MW}_{\text {th }}$ \\
Coolant mass flow rate & 25984 & $\mathrm{~kg} \mathrm{~s}^{-1}$ \\
Total number of FAs & 171 & - \\
Pins per FA & 127 & - \\
Coolant inlet temperature & 400 & ${ }^{\circ} \mathrm{C}$ \\
Coolant outlet temperature & 480 & ${ }^{\circ} \mathrm{C}$ \\
Vessel inner diameter & 3.4 & $\mathrm{~m}$ \\
FA width & 0.178 & $\mathrm{~m}$ \\
\hline
\end{tabular}




\section{Modelling approach}

The aim of a reduced order model is to replace a high-fidelity model, i.e., the Full Order Model (FOM), by one featuring a much lower computational complexity and retaining a comparable accuracy (Chinesta et al., 2016; Quarteroni et al., 2016). In the first step of the procedure, the highfidelity model is used to obtain different solutions of the considered problem for a properly selected number of instances of the input parameters. This information will be used to build a model able to perform low-cost real-time simulations for new instances of the parameter (Manzoni et al., 2012). The reduced solution is usually expressed as a linear combination of basis functions that are obtained starting from the snapshots (i.e., the solutions of the full order model). The coefficients of this combination are calculated by means of a Galerkin projection of the governing equations onto the reduced space. To this aim, test functions are also needed. In order to make the reduced order model efficient, the expensive computation of snapshots and the basis calculation can be performed just once (a demanding offline phase) and can be totally decoupled from the fast-running ROM simulation (an inexpensive online phase).

This modelling approach is applied to create a reduced order model for the neutronics of the ALFRED reactor (Figure 3). An offline procedure is needed to solve the neutron diffusion PDEs necessary for the calculation of the spatial basis and the test functions which are employed in the spatial neutronics model. In the following Sections, the spatial neutronics model and the methods for the creation of the basis and test functions are presented.
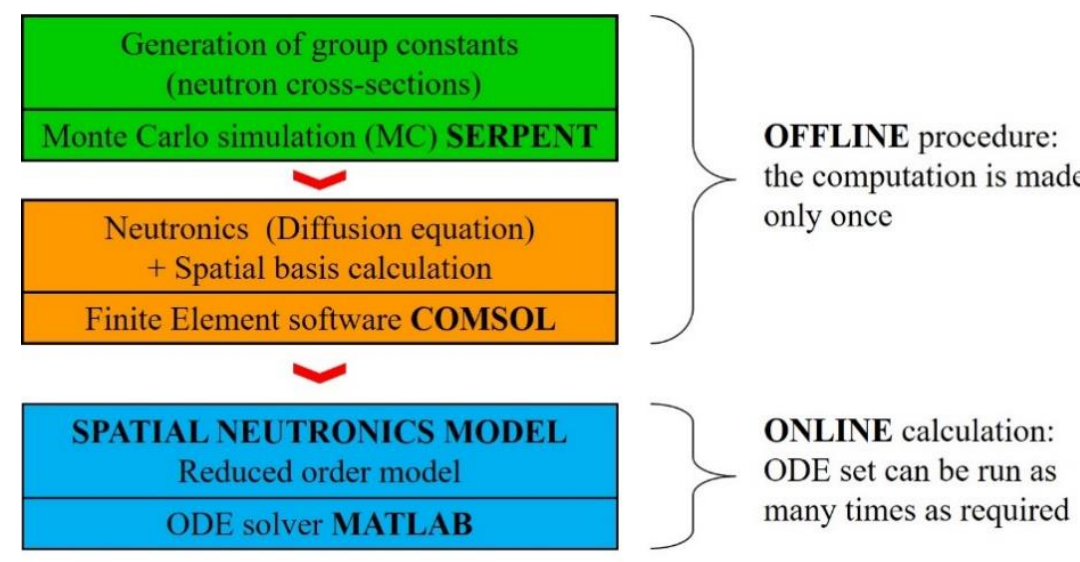

Figure 3. Derivation of the spatial neutronics model: offline procedure and online calculation. The offline procedure is made up by the generation of group constants using the SERPENT code and the calculation of the spatial basis (and test functions) using the multigroup diffusion equation implemented in COMSOL software. The online calculation, i.e., the reduced order model, can be easily implemented in an ODE solver.

\subsection{The spatial neutronics model}

For the derivation of the spatial neutronics model, the multi-group diffusion theory (Duderstadt and Hamilton, 1976), with $G$ energy groups and eight groups of precursors, is used as high-fidelity model. In equations, it reads:

$$
\underline{V}^{-1} \frac{\partial \underline{\phi}}{\partial t}=\nabla \cdot(\underline{\underline{D}} \nabla \underline{\phi})-\underline{\underline{\Sigma_{a}}} \underline{\underline{\phi}}-\underline{\underline{\Sigma_{s}}} \underline{\phi}+(1-\beta) \underline{\chi_{p}} \underline{F}^{T} \underline{\phi}+\sum_{j} \lambda_{j} \underline{\chi_{d}} C_{j}
$$




$$
\frac{\partial C_{j}}{\partial t}=-\lambda_{j} C_{j}+\beta_{j} \underline{F}^{T} \underline{\phi} \quad j=1 \div 8
$$

where

$$
\begin{gathered}
\underline{\phi}(\boldsymbol{r}, t)=\left[\begin{array}{c}
\phi_{1}(\boldsymbol{r}, t) \\
\vdots \\
\phi_{G}(\boldsymbol{r}, t)
\end{array}\right] \quad \phi_{g}(\boldsymbol{r}, t)=\int_{E_{g}}^{E_{g-1}} d E \phi(\boldsymbol{r}, E, t) \\
\underline{\underline{V}^{-1}}=\operatorname{diag}\left(\frac{1}{v_{g}}(\boldsymbol{r})\right) \quad \underline{\underline{D}}=\operatorname{diag}\left(D_{g}(\boldsymbol{r})\right) \quad \underline{\underline{\Sigma_{a}}}=\operatorname{diag}\left(\Sigma_{a}^{g}(\boldsymbol{r})\right) \\
\underline{\underline{\Sigma_{s}}}=\left[\begin{array}{cccc}
\Sigma_{s}^{1 \rightarrow(r)} & -\Sigma_{s}^{2 \rightarrow 1}(\boldsymbol{r}) & \cdots & -\Sigma_{s}^{G \rightarrow 1}(\boldsymbol{r}) \\
-\Sigma_{s}^{1 \rightarrow 2}(\boldsymbol{r}) & \Sigma_{s}^{2 \rightarrow}(\boldsymbol{r}) & \cdots & -\Sigma_{s}^{G \rightarrow 2}(\boldsymbol{r}) \\
\vdots & \vdots & \ddots & \vdots \\
-\Sigma_{s}^{1 \rightarrow G}(\boldsymbol{r}) & -\Sigma_{s}^{2 \rightarrow G}(\boldsymbol{r}) & \cdots & \Sigma_{s}^{G \rightarrow}(\boldsymbol{r})
\end{array}\right] \\
\underline{\chi_{p}}=\left[\begin{array}{c}
\chi_{p}^{1} \\
\vdots \\
\chi_{p}^{G}
\end{array}\right] \quad \underline{\chi_{d}}=\left[\begin{array}{c}
\chi_{d}^{1} \\
\vdots \\
\chi_{d}^{G}
\end{array}\right] \quad \underline{F}^{T}=\left[\begin{array}{lll}
v \Sigma_{f}^{1}(\boldsymbol{r}) & \cdots & \left.v \Sigma_{f}^{G}(\boldsymbol{r})\right]
\end{array}\right.
\end{gathered}
$$

The neutron flux can be expressed as

$$
\begin{gathered}
\underline{\phi}(\boldsymbol{r}, t) \cong \sum_{i=1}^{N} \underline{\psi}_{i}(\boldsymbol{r}) \cdot \underline{n}_{i}(t) \\
\underline{\psi_{i}}(\boldsymbol{r})=\left[\begin{array}{ccc}
\psi_{i}^{1}(\boldsymbol{r}) & 0 & 0 \\
0 & \ddots & 0 \\
0 & 0 & \psi_{i}^{G}(\boldsymbol{r})
\end{array}\right]=\operatorname{diag}\left(\underline{\psi}_{i}(\boldsymbol{r})\right) \\
\underline{n}_{i}(t)=\left[\begin{array}{c}
n_{i}^{1}(t) \\
\vdots \\
n_{i}^{G}(t)
\end{array}\right]
\end{gathered}
$$

with $\underline{\underline{\psi}}_{i}(\boldsymbol{r})$ the spatial basis where the flux is projected and $\underline{n}_{i}(t)$ the time-dependent coefficients.

A set of ODEs (whose unknowns are the time-dependent coefficient $\underline{n}_{i}(t)$ ) can be derived from the multi-group diffusion equations performing a Petrov-Galerkin projection. In particular, the Equation (4) has to be substituted into Equations (1) and (2), the latter have to be multiplied by test functions $\underline{\xi}_{i}=\operatorname{diag}\left(\xi_{i}(\boldsymbol{r})\right)$ and integrated over the computational domain. Finally, the ODE system for the timedependent coefficients can be expressed, for each basis function, as

$$
\begin{aligned}
& \sum_{m=1}^{N} \underline{\underline{R V_{i m}}} \cdot \underline{\underline{n}}_{m}=\sum_{m=1}^{N}\left(-\underline{\underline{L}}_{i m}-\underline{\underline{\delta}} \underline{\underline{\underline{L}}}+(1-\beta) \cdot\left(\underline{\underline{M_{i m}}}+\underline{\underline{\delta M_{i m}}}\right)\right) \cdot \underline{\underline{n}}_{m}+\sum_{j=1}^{8} \lambda_{j} \underline{c}_{i j} \\
& \dot{\underline{c}}_{i j}=\beta_{j} \underline{\underline{X}}\left[\sum_{m=1}^{N}\left(\underline{\underline{M_{i m}}}+\underline{\underline{\delta M_{i m}}}\right) \cdot \underline{n}_{m}\right]-\lambda_{j} \underline{c}_{i j} \quad j=1 \div 8
\end{aligned}
$$

where $\underline{\underline{L}}_{i m}$ and $\underline{\underline{M}}_{i m}$ represent the contribution to the removal and production operator calculated in the unperturbed system, and $\underline{\underline{\delta L}} i m$ and $\underline{\underline{\delta M_{i}}}$ represent the variation of the removal and the production operators during the transients. In Equations, it reads:

$$
\begin{gathered}
\underline{\underline{R V_{i m}}}=\int \underline{\underline{\xi_{i}}} \cdot \underline{\underline{V}}^{-1} \cdot \underline{\underline{\psi_{m}}} d \Omega \\
\underline{\underline{L_{i m}}}=\int \underline{\underline{\xi_{i}}} \cdot\left(-\nabla \cdot \underline{\underline{D}} \nabla+\underline{\underline{\underline{\Sigma_{a}}}}+\underline{\underline{\Sigma_{s}}}\right) \underline{\underline{\underline{\psi_{m}}}} d \Omega
\end{gathered}
$$




$$
\begin{aligned}
& \underline{\underline{\delta L}} i m=\int \underline{\underline{\xi_{i}}} \cdot \delta\left(-\nabla \cdot \underline{\underline{D}} \nabla+\underline{\underline{\Sigma_{a}}}+\underline{\underline{\Sigma_{s}}}\right) \underline{\underline{\psi_{m}}} d \Omega \\
& \underline{\underline{M_{i m}}}=\int \underline{\underline{\xi_{i}}} \cdot\left(\underline{\chi_{p}} \underline{F^{T}}\right) \underline{\underline{\psi_{m}}} d \Omega \\
& \underline{\underline{\delta M_{i m}}}=\int \underline{\underline{\xi_{i}}} \cdot \delta\left(\underline{\chi_{p}} \underline{F^{T}}\right) \underline{\underline{\psi_{m}}} d \Omega \\
& \underline{c}_{i j}=\int \underline{\underline{\xi_{i}}} \cdot \underline{\underline{\chi_{d}}} \cdot C_{j} d \Omega \quad \underline{\underline{X}}=\left[\begin{array}{ccc}
\chi_{d}^{1} / \chi_{p}^{1} & 0 & 0 \\
0 & \ddots & 0 \\
0 & 0 & \chi_{d}^{G} / \chi_{p}^{G}
\end{array}\right]
\end{aligned}
$$

$\underline{\underline{L_{i m}}}$ and $\underline{\underline{M}}_{i m}$ are calculated once in the "offline" process, and are kept constant during the transient simulation. The variation of removal and production operators, i.e., $\underline{\underline{\delta L}} i m$ and $\underline{\underline{\delta M}} i m$, is usually due to temperature change of material (i.e., the reactivity thermal feedbacks) or due to the CR movement. The operator variation (which represents a reactivity variation) is multiplied by the spatial basis and test functions. This way, it is possible to take into account the spatial characteristics of the perturbation. This leads to an accurate estimation of the reactivity evolution with respect to the PK approach in which the reactivity is uniformly calculated over the system.

From Equation (9), it is possible to realize that the terms involved in the reduced order model described by Equations (7) and (8) are values obtained through integrals. In particular, the integrals of Equation (9) are calculated on the high-fidelity model, according to the integration scheme employed (i.e., if the full order model is based on Finite Element discretization, the integrals are summation according to the quadrature rule). For the calculation of $\underline{\underline{\delta}} i m$ and $\underline{\delta} \underline{\underline{ }} i m$, the region involved in the integral calculation is not the overall system as for $\underline{\underline{L}}_{i m}$ and $\underline{\underline{M}}_{i m}$ since the variation of the removal and production operator (due to temperature change or CR movement) is not homogenous over the system. To correctly reproduce this feature, the geometry is divided in coarse zone representing the regions over the integrals are taken. For each region, the reactivity insertion is weighted on the spatial basis functions and the test functions integrated over the zone, considering the operator variation constant. The latter assumption consists in considering the temperature constant inside the coarse zone and consequently the reactivity variation, in case of the reactivity feedback. In case of CR movement, this entails that an effective variation of removal or production over the coarse zone following the CR movement is considered. This way, the calculation of the integral between the spatial basis and the test functions over the zone is made only in the offline process and kept constant in the online simulation. Finally, this quantity is multiplied by the removal (or production) variation, which is temperature or CR position dependent (and therefore also time-dependent). According to this procedure, $\underline{\underline{\delta L}} i m$ can be expressed as follows:

$$
\begin{gathered}
\underline{\underline{\delta} \underline{L}_{i m}}\left(h_{C R}, T\right)=\int \underline{\underline{\xi_{i}}} \cdot \delta L\left(h_{C R}, T\right) \underline{\underline{\psi_{m}}} d \Omega= \\
=\sum_{g z} \int \underline{\underline{\xi_{i}}}=\left[-\nabla \cdot\left(\underline{\underline{D}}\left(h_{C R, g z}, T_{g z}\right) \nabla\right)+\underline{\underline{\Sigma_{a}}}\left(h_{C R, g z}, T_{g z}\right)+\underline{\underline{\Sigma_{s}}}\left(h_{C R, g z}, T_{g z}\right)\right] \underline{\underline{\psi_{m}}} d \Omega_{g z}= \\
=\sum_{g z}\left[\int \nabla \underline{\underline{\xi_{i}}} \cdot \underline{\underline{D}}\left(h_{C R, g z}, T_{g z}\right) \nabla \underline{\underline{\psi_{m}}} d \Omega_{g z}-\int_{\partial \Omega_{g z}} \underline{\underline{\xi_{i}}} \cdot\left(\boldsymbol{n} \cdot \underline{\underline{D}}\left(h_{C R, g z}, T_{g z}\right) \nabla \underline{\underline{\psi_{m}}}\right) d S_{g z}+\right. \\
\left.+\int \underline{\underline{\xi_{i}}} \cdot \underline{\underline{\Sigma_{a}}}\left(h_{C R, g z}, T_{g z}\right) \cdot \underline{\underline{\psi_{m}}} d \Omega_{g z}+\int \underline{\underline{\xi_{i}}} \cdot \underline{\underline{\Sigma_{s}}}\left(h_{C R, g z}, T_{g z}\right) \cdot \underline{\underline{\psi_{m}}} d \Omega_{g z}\right]=
\end{gathered}
$$




$$
\begin{aligned}
& =\sum_{g z}\left[\underline{\underline{D}}\left(h_{C R, g z}, T_{g z}\right) \int \underline{\nabla \xi_{i}} \cdot \nabla \underline{\underline{\psi_{m}}} d \Omega_{g z}+\underline{\underline{\Sigma_{a}}}\left(h_{C R, g z}, T_{g z}\right) \int \underline{\underline{\xi_{i}}} \cdot \underline{\underline{\psi_{m}}} d \Omega_{g z}+\right. \\
& \left.+\underline{\underline{\Sigma_{s}}}\left(h_{C R, g z}, T_{g z}\right) \int \underline{\underline{\xi_{i}}} \cdot \underline{\underline{\psi_{m}}} d \Omega_{g z}\right]+\gamma_{r} \int_{\partial \Omega_{r}} \underline{\underline{\xi_{i}}} \cdot \underline{\underline{\psi_{m}}} d S_{r}+\gamma_{a} \int_{\partial \Omega_{a}} \underline{\xi_{i}} \cdot \underline{\underline{\psi_{m}}} d S_{a}
\end{aligned}
$$

where the subscript gz indicates a generic zone (region) of the coarse mesh where the integrals are taken over. In particular, $h_{C R, g z}$ and $\mathrm{T}_{\mathrm{gz}}$ are the height of the $\mathrm{CR}$ and the temperature in the generic zone, respectively. In Equation (10), the Green's first identity is used obtaining, in the final form, the surface integrals on the radial $\partial \Omega_{\mathrm{r}}$ and axial boundary $\partial \Omega_{\mathrm{a}}$ of the domain.

In order to solve the neutron diffusion PDEs, the neutronic parameters $\left(\mathrm{V}^{-1}, \mathrm{D}, \Sigma_{\mathrm{a}}, \Sigma_{\mathrm{s}}, \chi_{\mathrm{p}}, \chi_{\mathrm{d}}, \mathrm{F}^{\mathrm{T}}\right)$ should be calculated, e.g., by means of a Monte Carlo neutron transport code. The cross sections are homogenized in the coarse zones to be used in the neutron diffusion PDEs. In particular, in order to derive the reactivity effects due to the temperature and the CR movement, the following dependence on temperature and CR position of the cross section is employed:

$$
\Sigma\left(h_{C R}, T\right)=\left[\Sigma_{0}\left(h_{C R}\right)+A \cdot \log \left(\frac{T}{T_{0}}\right)\right]
$$

As far as the boundary conditions are concerned, the albedo boundary conditions are imposed at the axial and radial boundaries of the model domain, namely:

$$
\boldsymbol{n} \cdot\left(D_{g} \nabla \phi_{g}\right)=-\gamma_{a} \phi_{g} \quad \boldsymbol{n} \cdot\left(D_{g} \nabla \phi_{g}\right)=-\gamma_{r} \phi_{g}
$$

\subsection{Spatial basis calculation and the Adjoint Proper Orthogonal Decomposition}

One of the main challenges of the reduced order methods is to find the desired compromise between the accuracy and the size of the model, i.e., the computational cost (Quarteroni et al., 2011). In this sense, the choice of the spatial basis $\underline{\psi}_{i}(\boldsymbol{r})$ (i.e., the selection of the functions used to expand the neutron flux) and the test functions $\underline{\underline{z}}_{i}(\boldsymbol{r})$ is crucial for the spatial neutronics model. An optimized couple of spatial basis and test functions ensures faster simulation and improved accuracy. In this work, for the selection of the spatial basis functions, two methods are undertaken, namely the Modal Method and the Proper Orthogonal Decomposition. As for the test functions, typically they are the same functions constituting the spatial basis (Sartori et al., 2014). On the other hand, there is no limitation in using functions different from the spatial basis ones. In this regard, the adjoint functions related to the spatial basis are the logical choice from both a neutronics - since they are related to the neutron importance - and mathematical - due to the bi-orthogonality property - point of view. In particular, in the spatial neutronics model presented in Section 3.1, a formulation for the global and local reactivity similar to the classic definition in transport theory (Henry, 1975) can be derived

$$
\rho=\frac{\underline{n}^{T} \cdot[-(\underline{\underline{L}}+\underline{\underline{\underline{\delta}}})+((1-\beta)+\beta \underline{\underline{X}})(\underline{\underline{M}}+\underline{\underline{\delta M}})] \cdot \underline{\underline{n}}}{\underline{n}^{T} \cdot[((1-\beta)+\beta \underline{\underline{X}})(\underline{\underline{M}}+\underline{\underline{\delta M}})] \cdot \underline{\underline{n}}}
$$

In this work, an "Adjoint Proper Orthogonal Decomposition" method is proposed which is aimed at combining the feature of the Proper Orthogonal Decomposition for the spatial basis functions and the adjoint function as test functions. This approach can be intended to some extent as the counterpart of what presented in (Bang et al., 2012b) but applied to the projection-based framework (instead of 
the interpolation-based one). In order to assess the capability of the APOD method, a comparison with other couples of spatial basis functions and test functions is performed. In particular, the four cases studied (summarized in Table 2) are:

- Case A. The Modal Method is used for the creation of the spatial basis functions. The latter are also used as test functions. This method is adopted in (Sartori et al., 2014).

- Case B. The Modal Method is used for the creation of the spatial basis functions. As test functions, the adjoint functions related to the Modal Method are employed. This method is adopted in (Lorenzi et al., 2015).

- Case C. The POD is used for the creation of the spatial basis functions. The latter are also used as test functions. This method is adopted in (Sartori et al., 2014).

- APOD method (Case D). The POD is used for the creation of the spatial basis functions. As test functions, the adjoint functions related to the POD are employed. This method is proposed in this paper.

In the following, a brief description of the several methods is given.

Table 2. Spatial basis and test function pairs studied in this work.

\begin{tabular}{lllc}
\hline & & \multicolumn{2}{c}{ Test function } \\
\cline { 3 - 4 } & & Function of spatial basis & Adjoint functions \\
\hline \multirow{2}{*}{ Method for the spatial basis } & $M M$ & Case A (Sartori et al., 2014) & Case B (Lorenzi et al., 2015) \\
& $P O D$ & Case C (Sartori et al., 2014) & APOD (present work) - Case D \\
\hline
\end{tabular}

\subsubsection{Modal Method (Cases A and B)}

In the MM, the spatial basis is constituted of the eigenfunctions related to the neutron diffusion equation. The eigenvalue problem is

$$
\left(-\nabla \cdot \underline{\underline{D}} \nabla+\underline{\underline{\Sigma_{a}}}+\underline{\underline{\Sigma_{s}}}\right) \underline{\psi_{i}}=\lambda_{i}^{*} \underline{\chi_{t}} \underline{F}^{T} \underline{\psi}_{i}
$$

where the first eigenfunction $\psi_{1}$ gives the fundamental flux distribution and the smallest eigenvalue $\lambda^{*}{ }_{1}$ determines the core criticality condition. In this case, the spatial basis set is formed by functions referring to a unique reference configuration, i.e., the fundamental and the high-order eigenfunctions. As suitable test functions, both the same eigenfunctions (Case A) or the adjoint eigenfunctions of the Equation (14) (Case B) have been evaluated.

\subsubsection{Proper Orthogonal Decomposition and Adjoint Proper Orthogonal Decomposition (Cases C and $D)$}

Another possible option for the spatial basis $\underline{\underline{\psi}}_{i}(\boldsymbol{r})$ is the Proper Orthogonal Decomposition (POD) combined with the snapshot method (Holmes et al., 1996; Sirovich, 1987). The POD was initially used to analyse experimental data with a view to extracting dominant features and trends - in particular coherent structures (Berkooz et al., 1993). In the ROM framework, the POD is used to provide a "relevant" set of basis functions that allows identifying a low-dimensional subspace. Starting from selected numerical solutions (the snapshots) of the Full Order Model, i.e., the neutron diffusion PDEs, an optimal orthonormal POD basis is built that can be used for the reduced order model (i.e., the spatial neutronics model). The optimality feature means that the POD basis describes 
the snapshots better than any other basis. In addition, the first modes have the property to retain most of the information present in the original solutions (Berkooz et al., 1993). For a comprehensive introduction to POD, the reader may refer to (Holmes et al., 1996).

In order to obtain the POD basis, two main procedures are usually adopted, namely the Singular Value Decomposition (SVD) and the Correlation Matrix method (Volkwein, 1999). As for the spatial neutronics modelling, the SVD method is selected. The offline spatial basis calculation is the following:

1. Compute the $\mathrm{N}_{\mathrm{s}}$ snapshots $\phi^{1}, \phi^{2}, \ldots, \phi^{N s}$;

2. Build the matrix of the snapshots $\underline{\underline{Y}}=\left[\phi^{1}, \phi^{2}, \ldots, \phi^{N s}\right]$;

3. Perform the SVD on $\underline{\underline{Y}}$ in order to obtain:

$$
\underline{\underline{Y}}=\underline{\underline{U}} \underline{\underline{S}} \underline{\underline{G}}^{T}
$$

where $\underline{U}=\left[\underline{\psi}_{1}{ }^{P O D}, \underline{\psi}_{2}{ }^{P O D}, \ldots, \underline{\psi}_{N p}^{P O D}\right]$ is the matrix containing the POD modes $\underline{\psi}_{i}^{P O D}, \underline{\underline{S}}=\operatorname{diag}\left(\sigma_{i}\right)$ is the matrix containing the singular values $\sigma_{i}$ associated to each mode and sorted in descending order of relevance. A high value of the singular value indicates that the related mode contributes in a significant way to the reconstruction of information contained in the snapshots. The snapshots are collected from the fundamental eigenfunctions related to different configuration of the ALFRED reactor in terms of temperature and CR position.

As suitable test functions, the same POD modes are usually employed (Case C). On the other hand, other options can be considered. The idea of an Adjoint Proper Orthogonal Decomposition lays on the fact that in the neutronics field, and in particular in the transport field, the adjoint flux is used as test function in the determination of the reactivity insertion during a variation of production or removal operator (Henry, 1975). For the calculation of the spatial basis functions, the procedure is the same of the POD which led to Equation (15). For the test functions, the following procedure is proposed:

1. Compute the $\mathrm{N}_{\mathrm{s}}$ adjoint snapshots $\phi^{\dagger, 1}, \phi^{\dagger, 2}, \ldots, \phi^{\dagger, N s}$ related to the same configuration of the snapshot calculated for the POD modes;

2. Build the matrix of the adjoint snapshots $\underline{\underline{A}}=\left[\phi^{\dagger, 1}, \phi^{\dagger, 2}, \ldots, \phi^{\dagger, N s}\right]$;

3. Compute the matrix of test functions $\underline{\underline{E}}$ starting from

$$
\underline{\underline{A}}=\underline{\underline{E}} \underline{\underline{S}} \underline{\underline{G}}^{T}
$$

where $\underline{\underline{E}}=\left[\xi_{1}{ }^{A P O D}, \underline{\xi}_{2}{ }^{A P O D}, \ldots, \underline{\xi}_{N p}{ }^{A P O D}\right]$ is the matrix containing the test function modes $\underline{\xi}_{i}^{A P O D}, \underline{\underline{\mathrm{S}}}$ and $\underline{\underline{G}}$ are the matrices obtained from the SVD of the spatial basis (see Equation (15)). Instead of performing a new SVD, test functions (i.e., the E matrix) are calculated inverting the Equation (16). In this way, since the spatial and test functions are linear combination of the snapshots (Volkwein, 1999), the test functions are obtained according to the same "decomposition" of the spatial basis, i.e., they share the same coefficients of the linear combination used to obtain the spatial basis functions. This modelling choice was preferred to a completely new SVD since, in the latter case, the correlation between direct and adjoint flux would be lost in the new SVD calculation, giving a different linear combination, and hence prioritization, in the construction of the test functions creation. 


\section{Simulation results}

The performance of the several methods proposed in Section 3 has been assessed. To this aim, a model of the entire core of the ALFRED reactor at Fuel Assembly (FA) (Figure 2) level has been developed. In particular, the focus is kept on the capability of the different pairs of spatial basis/test functions to correctly reproduce the reactivity due to the thermal feedback and the CR movements.

\subsection{The offline procedure}

A detailed model of ALFRED is set up with a heterogeneous description of the reactor using the continuous energy Monte Carlo neutron transport code SERPENT (Leppänen et al., 2015), which features group constant generation capabilities. The SERPENT model represents the 171 FAs, 110 dummy elements, 12 CRs and 4 SRs, the inner vessel and the surrounding lead (Figure 4a). The zones above and below the active zone are also modelled (Figure 4b) in order to take into account the CRs and SRs position as well as the contribution of these zones to the reactivity effect (i.e., in particular for the lead density). For the isotopic composition of the input materials concerning ALFRED (fuel, cladding, coolant, control rods), the reader may refer to (Grasso et al., 2014). The group constants of the seven energy groups (Table 3) are obtained after runs of 150 million active neutron histories.

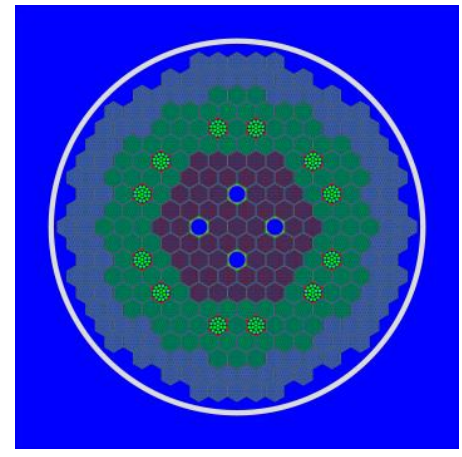

(a)

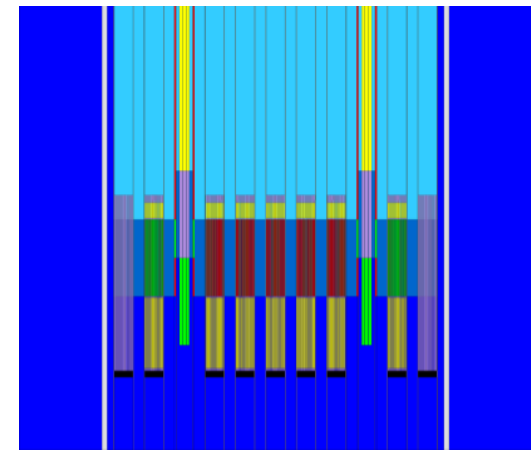

(b)

Figure 4. Radial view (a) and longitudinal view (b) of the SERPENT model of the ALFRED reactor. It is possible to notice $171 \mathrm{FAs}, 110$ dummy elements, $12 \mathrm{CRs}$ and $4 \mathrm{SRs}$, the inner vessel. In the axial direction, the zone above and below the core are modelled to consider the CR movement.

Several simulations have been carried out at different conditions to derive the main reactivity feedback effects of the ALFRED reactor and the CR insertion curve, using the nuclear data library JEFF 3.1 (Koning, 2006). The results of the thermal reactivity coefficients are summarized in Table 4, and compared to the outcomes of the LEADER Project calculated by means of the deterministic transport code ERANOS (Grasso et al., 2014). As for the CR insertion, several SERPENT simulations at different $\mathrm{CR}$ positions have been performed. The reactivity curve of the control rods is shown in Figure 5.

Table 3. Energy group boundaries for the generation of the group constant to be used for the creation of the group constants in SERPENT (using the nuclear data library JEFF 3.1).

\begin{tabular}{ccc}
\hline Group & Upper boundary & Lower boundary \\
\hline 1 & $20 \mathrm{MeV}$ & $2.23 \mathrm{MeV}$ \\
2 & $2.23 \mathrm{MeV}$ & $0.82 \mathrm{MeV}$ \\
3 & $0.82 \mathrm{MeV}$ & $0.30 \mathrm{MeV}$ \\
4 & $0.30 \mathrm{MeV}$ & $67.38 \mathrm{keV}$ \\
5 & $67.38 \mathrm{keV}$ & $15.03 \mathrm{keV}$ \\
6 & $15.03 \mathrm{keV}$ & $0.75 \mathrm{keV}$ \\
7 & $0.75 \mathrm{keV}$ & $0 \mathrm{keV}$ \\
\hline
\end{tabular}


Table 4. Comparison of the reactivity coefficients (end of cycle) of the ALFRED reactor for different reactivity effect (Doppler, lead expansion, axial fuel expansion, axial cladding expansion, axial wrapper expansion, radial grid expansion). The results are in good agreement with deterministic calculation performed with ERANOS code (Grasso et al., 2014).

\begin{tabular}{lcc}
\hline & $\begin{array}{c}\text { SERPENT } \\
\text { (present work) }\end{array}$ & $\begin{array}{c}\text { ERANOS } \\
\text { (Grasso et al., 2014) }\end{array}$ \\
\hline Doppler constant $(\mathrm{pcm})$ & $-549 \pm 18$ & -566 \\
Lead expansion coefficient $(\mathrm{pcm} / \mathrm{K})$ Case $1^{\dagger}$ & $-0.327 \pm 0.019$ & - \\
Lead expansion coefficient $(\mathrm{pcm} / \mathrm{K})$ Case $2^{\ddagger}$ & $-0.268 \pm 0.019$ & -0.268 \\
Axial fuel expansion $(\mathrm{pcm} / \mathrm{K})$ & $-0.152 \pm 0.006$ & -0.155 \\
Axial cladding expansion $(\mathrm{pcm} / \mathrm{K})$ & $+0.044 \pm 0.006$ & +0.039 \\
Axial wrapper expansion $(\mathrm{pcm} / \mathrm{K})$ & $+0.036 \pm 0.006$ & +0.023 \\
Radial grid expansion $(\mathrm{pcm} / \mathrm{K})$ & $-0.780 \pm 0.007$ & -0.789 \\
\hline
\end{tabular}

$\dagger$ Calculated considering all the lead inside the inner vessel.

† Calculated for the whole height of the fissile subassemblies.

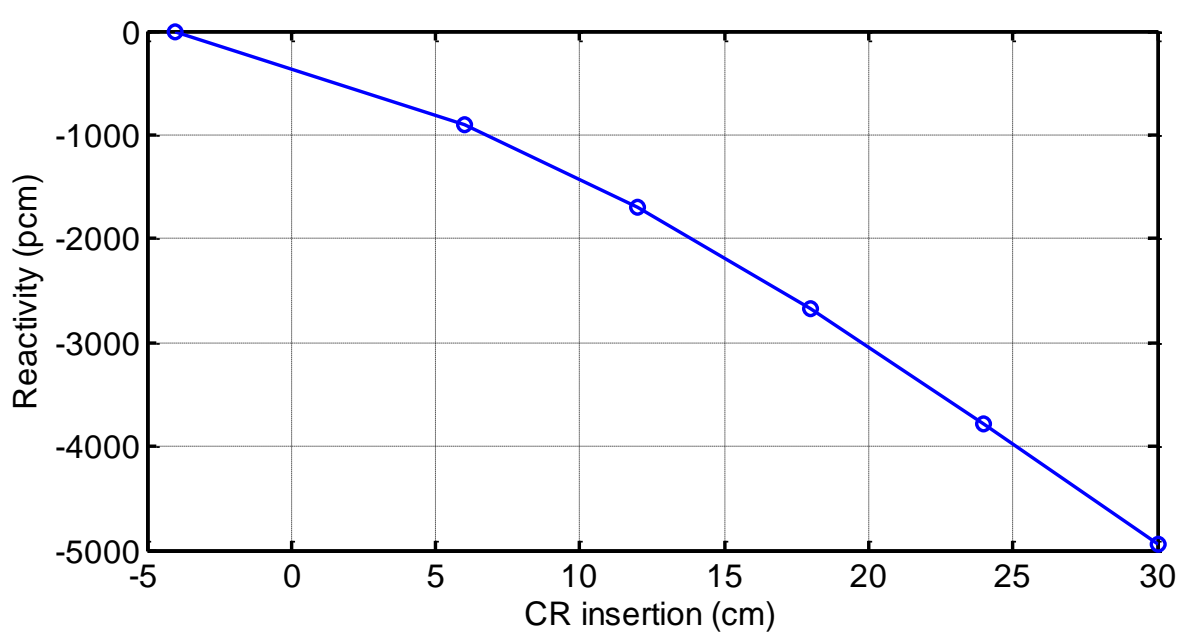

Figure 5. Reactivity curve of the control rods (calculated by SERPENT - statistical errors are below 10 pcm and were omitted from the plot). Negative $C R$ insertion indicates that the $C R$ is extracted (the negative value is the distance of the CR from the active zone).

In order to obtain the spatial basis and the test functions for the different case (MM with and without adjoint, POD and APOD - see Table 2), the eigenvalue problem of the neutron diffusion PDEs and its adjoint are solved adopting the Finite Element COMSOL Multiphysics software (COMSOL, 2011) in different configurations of the ALFRED core in terms of temperature values and CR position. The mesh of the ALFRED core model (Figure 6) features a 3D geometry using Lagrangian and linear-order elements (92664 triangular elements and 57240 hexahedral elements for a total of 213840 prism elements). The mesh quality is very good, reaching a minimum and average element of 0.8935 and 0.9296 , respectively. The cross-sections calculated by means of SERPENT are homogenized in different zones (Figure 7), i.e., 5 for the inner fuel, 5 for the outer fuel, 4 for the SRs, 5 for the CRs and 3 for the dummy elements. As far as the boundary conditions are concerned, the albedo boundary conditions, previously calculated in the SERPENT model, are imposed at the axial and radial boundaries of the COMSOL model domain. The Implicitly Restarted Arnoldi Method (an Arnoldi-like eigenvalue solver (Sorensen, 2002)) is adopted to solve the eigenvalue problem necessary for the spatial basis and test functions. Starting from the solutions of the eigenvalue problem, the SVD routine of MATLAB toolkit (MATLAB, 2016) and ad-hoc developed routine 
implementing the procedure described in the Section 3.2.2 for the APOD method are used to derive the spatial basis and test functions of Case C and APOD method.

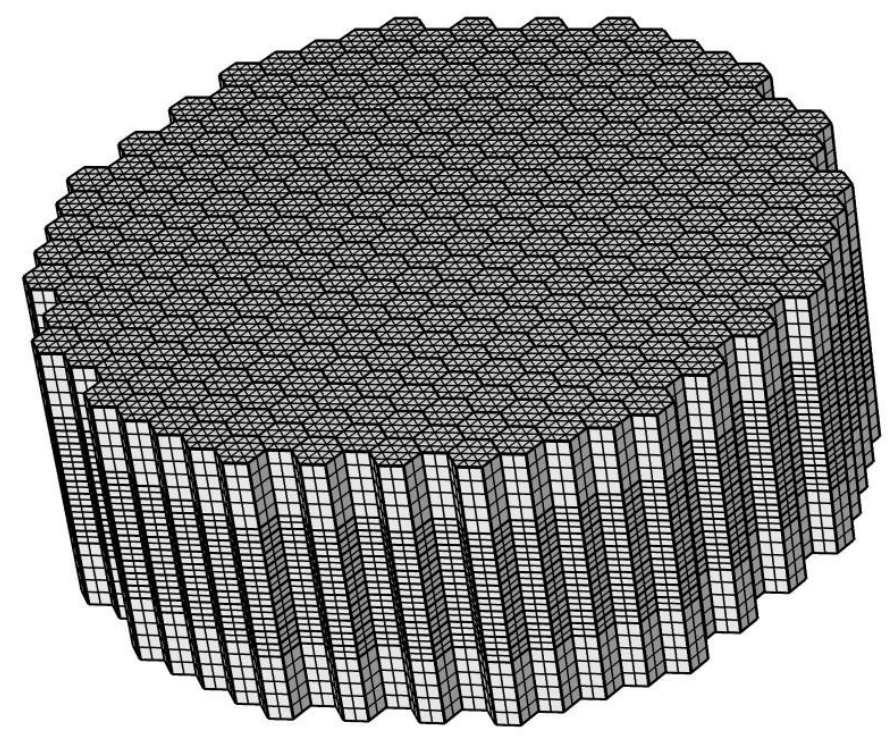

Figure 6. Mesh of the COMSOL model of the ALFRED reactor. The computational grid is made of using Lagrangian and linear-order elements (92664 triangular and 57240 hexahedral) for a total of 213840 prism elements). Each FA is divided in 24 elements in the radial direction and 20 elements in axial direction.

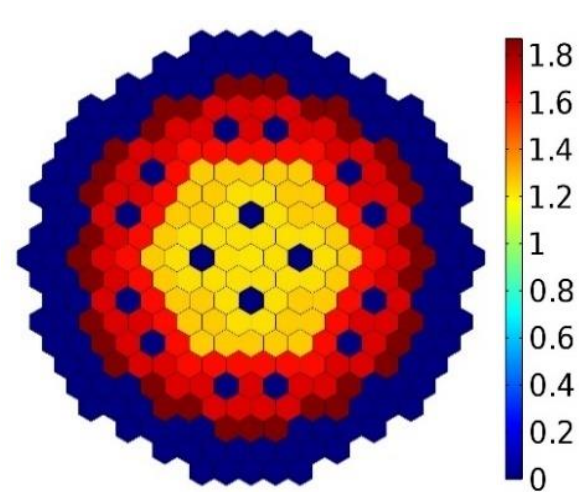

(a)

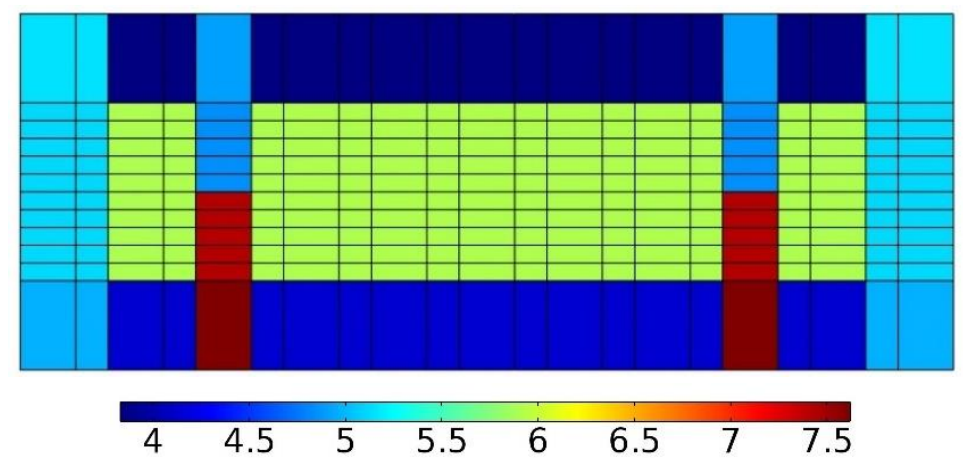

(b)

Figure 7. Example of the cross sections discretization. (a) Homogenized fission cross-section $\left(\mathrm{m}^{-1}\right)$ for the $7^{\text {th }}$ energy group. The fission cross section is zero in the dummy elements and in the CRs and SRs positions. (b) Removal cross-section $\left(\mathrm{m}^{-1}\right)$ for the $1^{\text {st }}$ energy group, employed in the COMSOL model. The high value of the removal cross section represents the insertion of the $\mathrm{CR}$ at half core.

\subsection{Spatial neutronics model - thermal feedback results}

The capability of the spatial neutronics model in the correct estimation of the temperature reactivity feedbacks has been assessed. The spatial basis and test functions of the MM (cases A and B) are calculated starting from the reference configuration (Figure 8), whereas seven simulation cases have been carried out at the conditions described in Table 5 for the POD and APOD (Figure 9, cases C and D). 
Table 5. Simulation cases carried out to reproduce the thermal reactivity feedbacks (Doppler, lead expansion, axial fuel expansion, radial grid expansion) and used for the snapshots generation. For the Doppler effect and the axial fuel expansion, variation in inner and outer fuel zones are treated separately.

\begin{tabular}{|c|c|c|c|c|c|}
\hline & $\begin{array}{l}\text { Fuel temp. (K) } \\
\text { (inner/outer) }\end{array}$ & $\begin{array}{l}\text { Lead temp. (K) } \\
\text { (below active zone } \\
\text { \& dummy/active } \\
\text { zone/above active } \\
\text { zone) }\end{array}$ & $\begin{array}{l}\text { Active length (cm) } \\
\text { (inner/outer) }\end{array}$ & $\begin{array}{l}\text { Fuel density }\left(\mathrm{g} \mathrm{cm}^{-3}\right) \\
\quad \text { (inner/outer) }\end{array}$ & FA pitch $(\mathrm{cm})$ \\
\hline Unperturbed & $1500 / 1200$ & $673 / 713 / 753$ & $60 / 60$ & $10.443 / 10.47$ & 17.1 \\
\hline Doppler (inner zone) & $900 / 1200$ & $673 / 713 / 753$ & $60 / 60$ & $10.443 / 10.47$ & 17.1 \\
\hline Doppler (outer zone) & $1500 / 600$ & $673 / 713 / 753$ & $60 / 60$ & $10.443 / 10.47$ & 17.1 \\
\hline Lead density & $1500 / 1200$ & $1473 / 1513 / 1573$ & $60 / 60$ & $10.443 / 10.47$ & 17.1 \\
\hline $\begin{array}{l}\text { Axial fuel expansion } \\
\text { (inner zone) }\end{array}$ & $1500 / 1200$ & $673 / 713 / 753$ & $61.2245 / 60$ & $10.234 / 10.47$ & 17.1 \\
\hline $\begin{array}{l}\text { Axial fuel expansion } \\
\text { (outer zone) }\end{array}$ & $1500 / 1200$ & $673 / 713 / 753$ & $60 / 61.2245$ & $10.443 / 10.261$ & 17.1 \\
\hline $\begin{array}{c}\text { Radial grid } \\
\text { expansion }\end{array}$ & $1500 / 1200$ & $673 / 713 / 753$ & $60 / 60$ & $10.443 / 10.47$ & 17.1855 \\
\hline
\end{tabular}
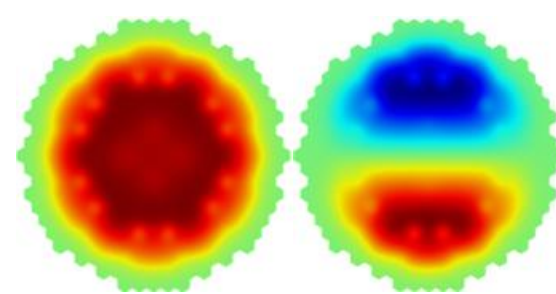

(a)

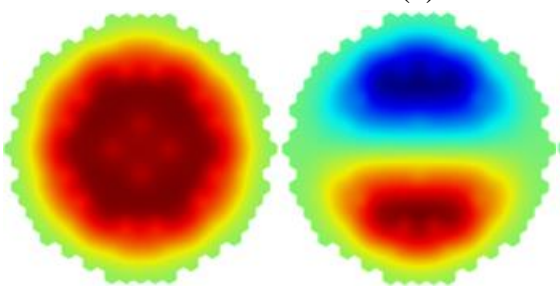

(c)
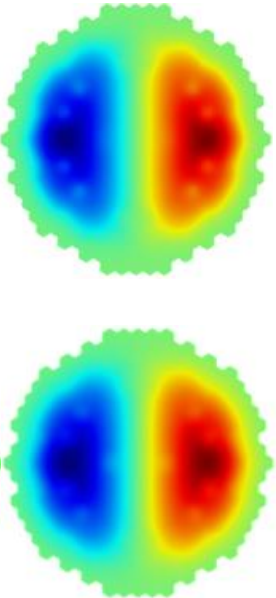
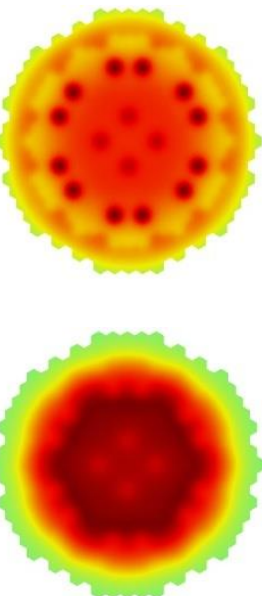
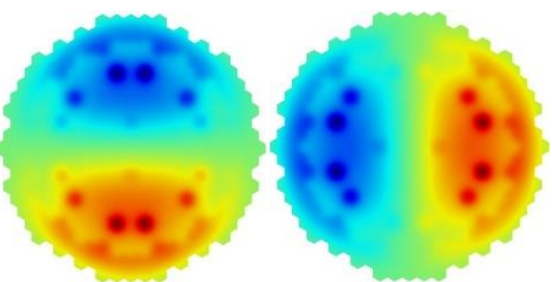

(b)

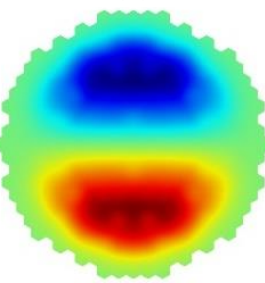

(d)

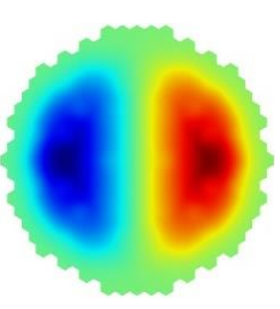

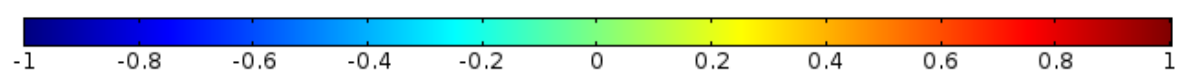

\section{.}




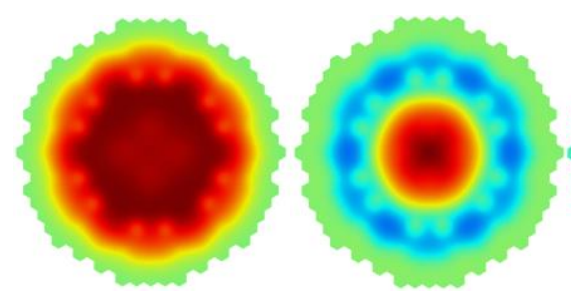

(a)
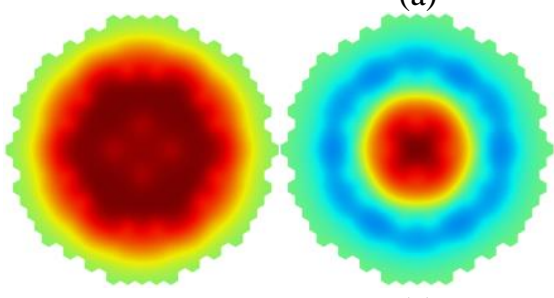

(c)
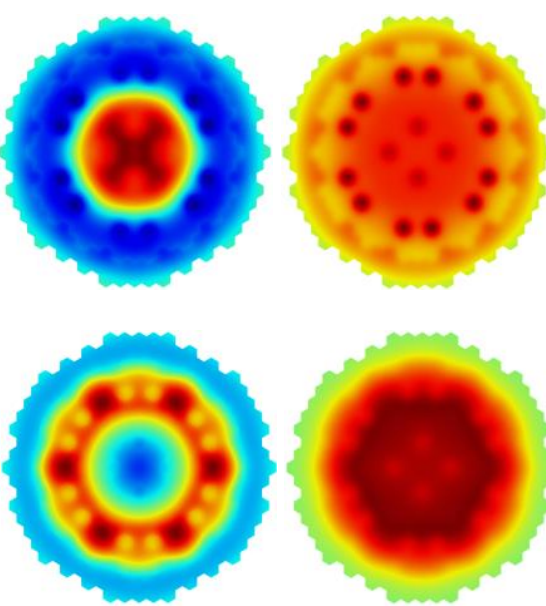

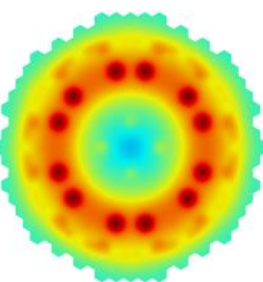

(b)

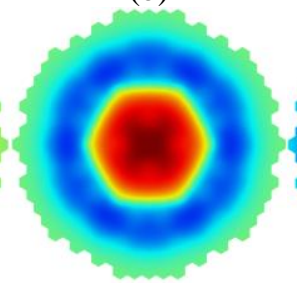

(d)
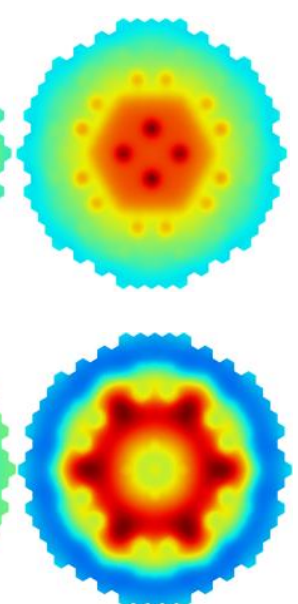

0.8

Figure 9. First three functions (at the midplane) of the spatial basis and test functions for the POD and APOD methods (Cases $C$ and D) - thermal reactivity effects. Spatial basis functions (Cases $C$ and $D$ ) and test functions (Case C) for (a) the third energy group and (b) the sixth energy group; test functions (Case D) for the (c) third energy group and (d) the sixth energy group (in normalized unit).

Table 6. Comparison of the SERPENT and COMSOL simulation results for the thermal reactivity feedbacks. The reactivity variation $(\mathrm{pcm})$ is taken as figure of merit to compare the results of the two modelling tools.

\begin{tabular}{cccc}
\hline & $\begin{array}{c}\text { SERPENT } \\
\text { (transport) }\end{array}$ & $\begin{array}{c}\text { COMSOL } \\
\text { (diffusion) }\end{array}$ & $\begin{array}{c}\text { Error } \\
(\%)\end{array}$ \\
\hline Unperturbed & - & - & - \\
Doppler (inner zone) & $128.1 \pm 7.5$ & 121.1 & 5.5 \\
Doppler (outer zone) & $206.7 \pm 7.5$ & 224.3 & 8.5 \\
Lead density & $-261.7 \pm 7.5$ & -275.3 & 5.21 \\
Axial fuel expansion (inner zone) & $-101.5 \pm 7.5$ & -105.6 & 4 \\
Axial fuel expansion (outer zone) & $-154.6 \pm 7.5$ & -139.1 & 10 \\
Radial grid expansion & $-206.8 \pm 7.5$ & -207 & 0.11 \\
\hline
\end{tabular}

The four pairs of spatial basis/test functions reported in Table 2 are implemented and their capability to reproduce the reactivity feedbacks is verified, considering from five to seven basis functions. The reactivity variation between the unperturbed case and the representative simulation of the temperature effect is assessed in terms of global features and spatial distribution in the reactor. For the sake of brevity, only the results regarding the Doppler (inner zone) and the lead density effects are reported in the following (Table 7). Nevertheless, the other four simulations, regarding the Doppler effect (outer zone), axial and radial expansion, show similar results and lead to the same conclusions. Five functions for the flux expansion are considered for the Cases A, B, C, D. In addition, the result employing seven functions for the Case $\mathrm{C}$ is given. The results indicate that the best performance is obtained in Case D (APOD method) and also in Case C (classic POD method) with seven expansion functions. Nevertheless, Case D reaches the same performance of Case C (i.e., correctly reproducing the insertion in the reference) with less functions. This means that, if employed in the spatial neutronics model, the set of ODEs based on APOD will require less computational time than the POD one. As far as the Modal Method is concerned, acceptable results are reached only if the adjoint eigenfunctions are employed as test functions (Case B). 
Table 7. Thermal feedbacks. Reactivity variation ( $\mathrm{pcm}$ ) calculated by the spatial neutronics model using the different methods presented in the paper (from five to seven spatial basis functions). The reference value is the reactivity calculated by the COMSOL model. Good results are obtained by the APOD method with a limited number of functions.

\begin{tabular}{lcccccc}
\hline & $\begin{array}{c}\text { Case A } \\
(\mathrm{N}=5)\end{array}$ & $\begin{array}{c}\text { Case B } \\
(\mathrm{N}=5)\end{array}$ & $\begin{array}{c}\text { Case C } \\
(\mathrm{N}=5)\end{array}$ & $\begin{array}{c}\text { Case C } \\
(\mathrm{N}=7)\end{array}$ & $\begin{array}{c}\text { Case D } \\
(\mathrm{N}=5)\end{array}$ & $\begin{array}{c}\text { COMSOL } \\
\text { (reference) }\end{array}$ \\
\hline Doppler - Inner zone & 46.7 & 119.1 & 98.6 & 121.1 & 121.1 & $\mathbf{1 2 1 . 1}$ \\
Lead density & -559.1 & -286.2 & -277.4 & -275.3 & -275.3 & $\mathbf{- 2 7 5 . 3}$ \\
\hline
\end{tabular}

The evolution of the reactivity versus the number of the functions employed ${ }^{1}$ is described in Figure 10a and Figure 10b for the Doppler effect (inner zone) and lead density effect, respectively. This analysis is useful to understand how many functions should be included for the flux expansion, giving a figure of merit for the employed methods. In particular, as far as the Modal Method is concerned (Case B), it is clear that only the first function contributes to the estimation of the reactivity variation, i.e., the dominant eigenfunction of the reference configuration. As for the POD and APOD methods, the convergence to the reference result is reached using three or four functions for the Case $\mathrm{D}$, whereas for the Case $\mathrm{C}$ different outcomes are obtained. In particular, for the lead density effect, an acceptable result for the Case $\mathrm{C}$ is obtained with three functions but, for the Doppler effect, relevant discrepancies are observed until the $7^{\text {th }}$ mode is added. Conversely, the APOD method is not affected by this dependence on the convergence to the reference value.

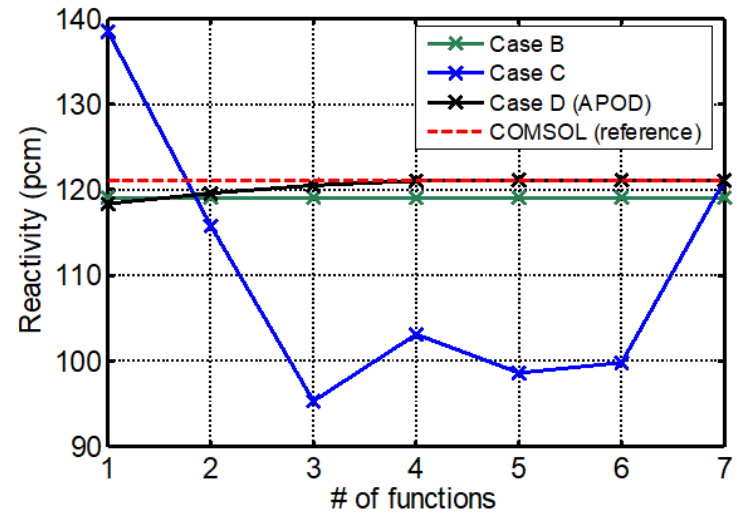

(a)

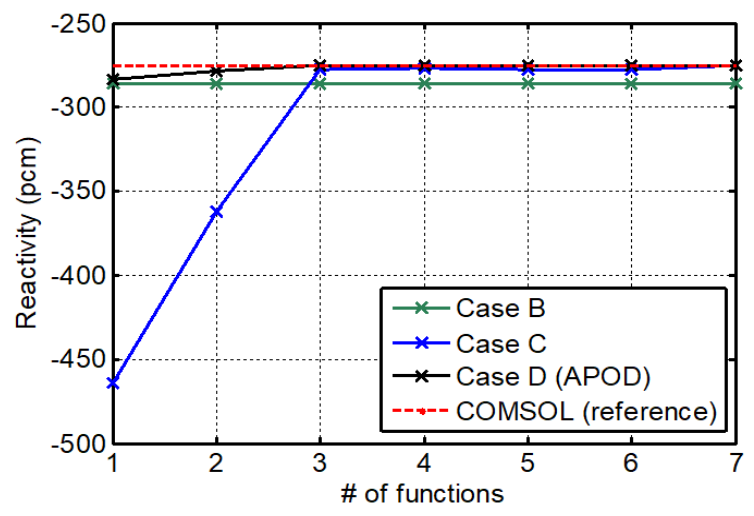

(b)

Figure 10. Doppler effect in inner fuel zone (a) and lead density effect (b). Evolution of the reactivity variation versus the number of functions employed in the model for the different methods used in the paper. Case $A$ is not reported since it is not suitable to reproduce the reactivity. The APOD method shows a better performance with respect the other methods.

One of the major advantage of employing the spatial neutronics model is the possibility to take into account the reactivity spatial distribution, i.e., the temperature feedbacks can be locally calculated, improving the model accuracy compared to the classic Point Kinetics. As already mentioned in the Introduction, the lead density effect may be positive or negative according to the zone involved. This spatial effect is taken into account in the spatial neutronics model as represented in Figure 11. In particular, the lead density effect is positive in the centre of the core where the effect of absorption reduction is predominant on the effect of the increase of leakage. On the other hand, the contribution is negative in the periphery of the active zone, i.e, in some FAs of the outer zone, in the dummy elements and in the upper and lower reflector. The possibility to take into account these effects in the dynamics modelling is definitely a major outcome of the spatial neutronics model.

\footnotetext{
${ }^{1}$ The Case A has been excluded from this analysis since it is not suitable to reproduce the reactivity.
} 


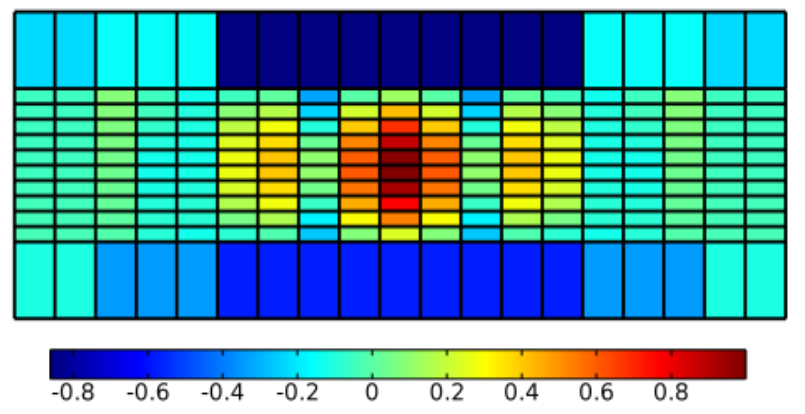

Figure 11. Lead density effect. Spatial reactivity variation (pcm) calculated with APOD method (Case D). The reactivity is estimated using Equation (13) for each zone of the coarse mesh. The lead density effect, as predicted by the method, is positive in the centre of the core and negative in the periphery.

The capability to reproduce different situations from what included in the snapshots has been assessed. Even if the optimum is to calculate as much as possible snapshots related to the system behaviour in order to "train" the spatial basis, it is not possible to include every possible situation that may happen in the core and therefore it is important to ensure acceptable results if the simulation is run in conditions different from the ones considered in the snapshots set. This can be achieved employing CRT method instead of SRS one. The reason relies on the fact that the first method is problem-dependent and the other one is problem-transparent. In particular, with CRT methods, the reduced order model is derived starting from a physical modelling rather than a fitting of input/output relationships. This way, the missing information due to the lack of snapshots can be partially substituted by the model-oriented structure of the reduced order model. In this work, as far as the lead density is concerned, only one case has been included in the snapshots, representing an increase of $800 \mathrm{~K}$ of the lead temperature in the entire system. For this reason, a simulation involving $800 \mathrm{~K}$ lead temperature increase only in the fuel region has been carried out. The reactivity results for the different cases are reported in Table 8. The results show that, for a situation which is not directly contemplated in the set of snapshots, the APOD method provides better results than POD given the reduced error compared to the reference result of COMSOL.

Table 8. Lead density effect in the fuel region (not trained in the snapshots). Reactivity variation (pcm) calculated by the spatial neutronics model using the different methods presented in the paper (from five to seven spatial basis functions). The reference value is the reactivity calculated by the COMSOL model. Good results are obtained by the APOD method with a limited number of functions.

\begin{tabular}{cccccc}
\hline $\begin{array}{c}\text { Case A } \\
(\mathrm{N}=5)\end{array}$ & $\begin{array}{c}\text { Case B } \\
(\mathrm{N}=5)\end{array}$ & $\begin{array}{c}\text { Case C } \\
(\mathrm{N}=5)\end{array}$ & $\begin{array}{c}\text { Case C } \\
(\mathrm{N}=7)\end{array}$ & $\begin{array}{c}\text { Case D } \\
(\mathrm{N}=5)\end{array}$ & $\begin{array}{c}\text { COMSOL } \\
\text { (reference) }\end{array}$ \\
\hline-56.2 & 3.9 & 54.9 & 31.8 & 7.8 & $\mathbf{8 . 7}$ \\
\hline
\end{tabular}

\subsection{Spatial neutronics model - CR movement results}

The spatial neutronics model has been tested in the estimation of the reactivity insertion due to the CR movement. The spatial basis and test functions of the MM (cases A and B) are the same of the thermal feedback study (Figure 8) due to the approach itself that relies on the high-order eigenfunctions calculated in a reference configuration. As for the POD and APOD methods, the spatial basis and test functions (Figure 12) are calculated starting from different cases at different CR position obtained with SERPENT and COMSOL simulations (see Figure 13), in particular the CR configurations shown in Table 9 are used for the snapshots generation. Up to seven basis functions are considered for the spatial neutronics model. 


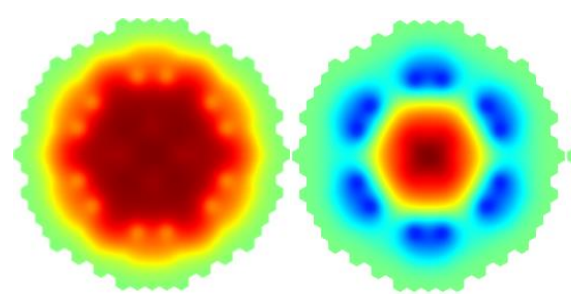

(a)
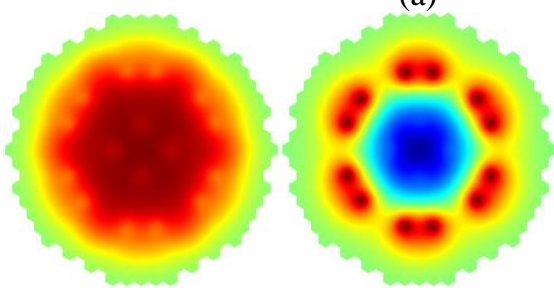

(c)
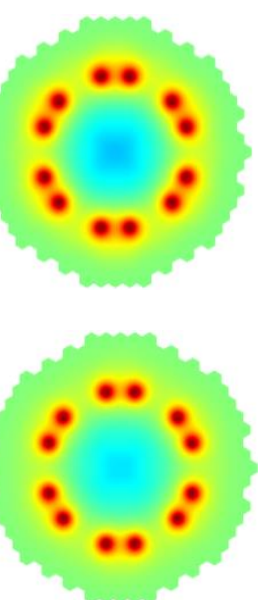
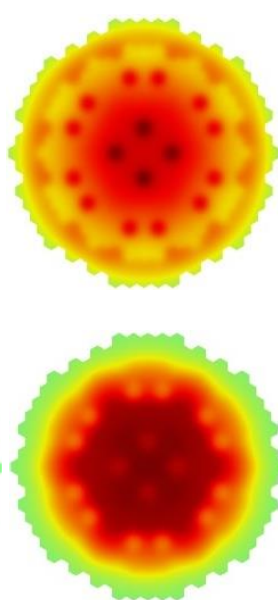

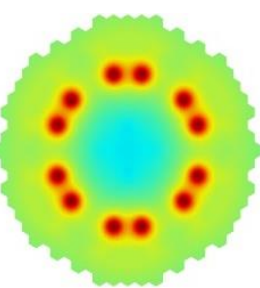

(b)

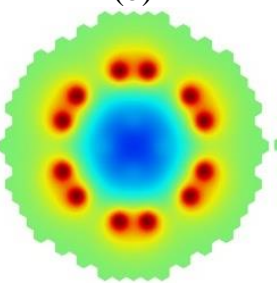

(d)
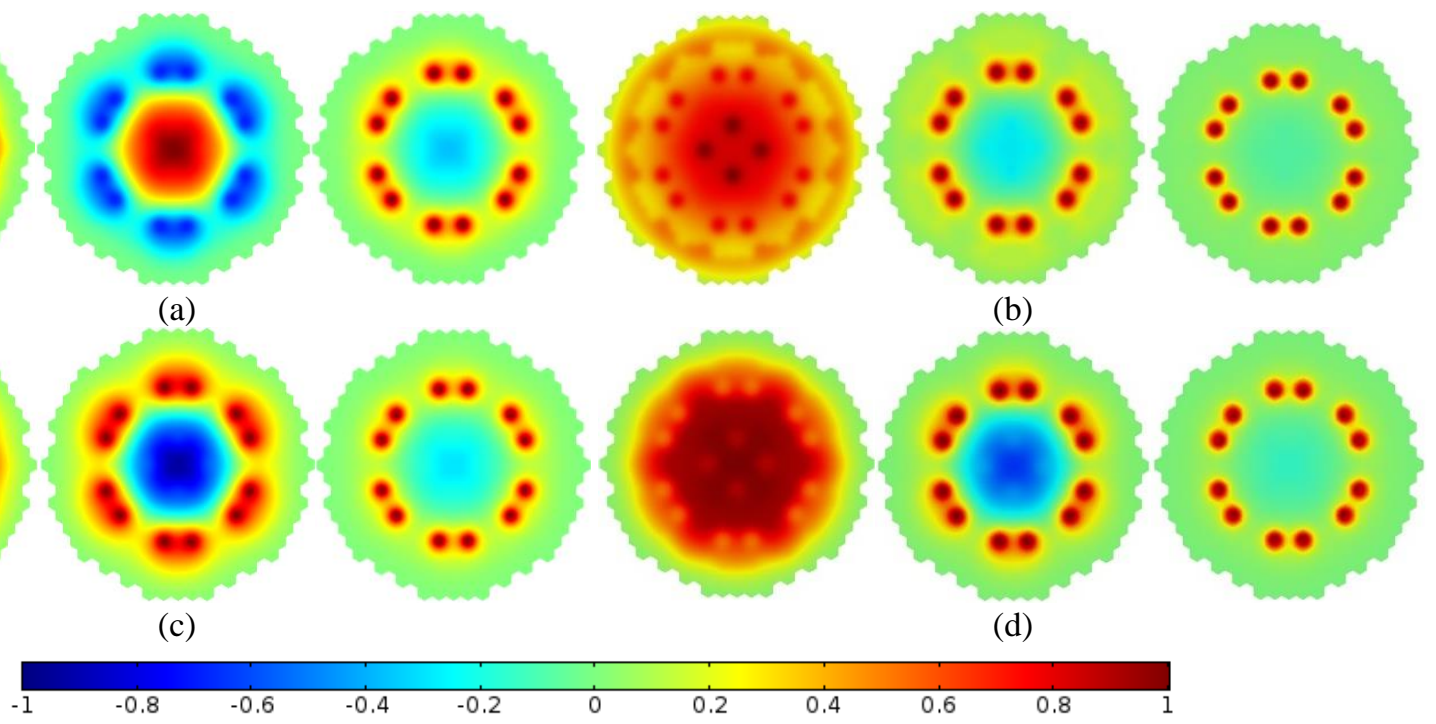

$\begin{array}{ll}-0.8 & -0.6\end{array}$

$-0.4$

0

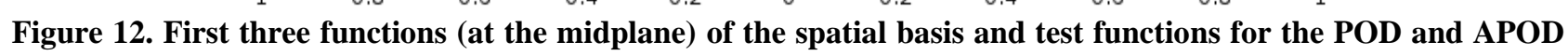

0.6

0.8 methods (Cases C and D) - CR movement. Spatial basis functions (Cases C and D) and test functions (Case C) for (a) the third energy group and (b) the sixth energy group; test functions (Case D) for the (c) third energy group and (d) the sixth energy group (in normalized unit).

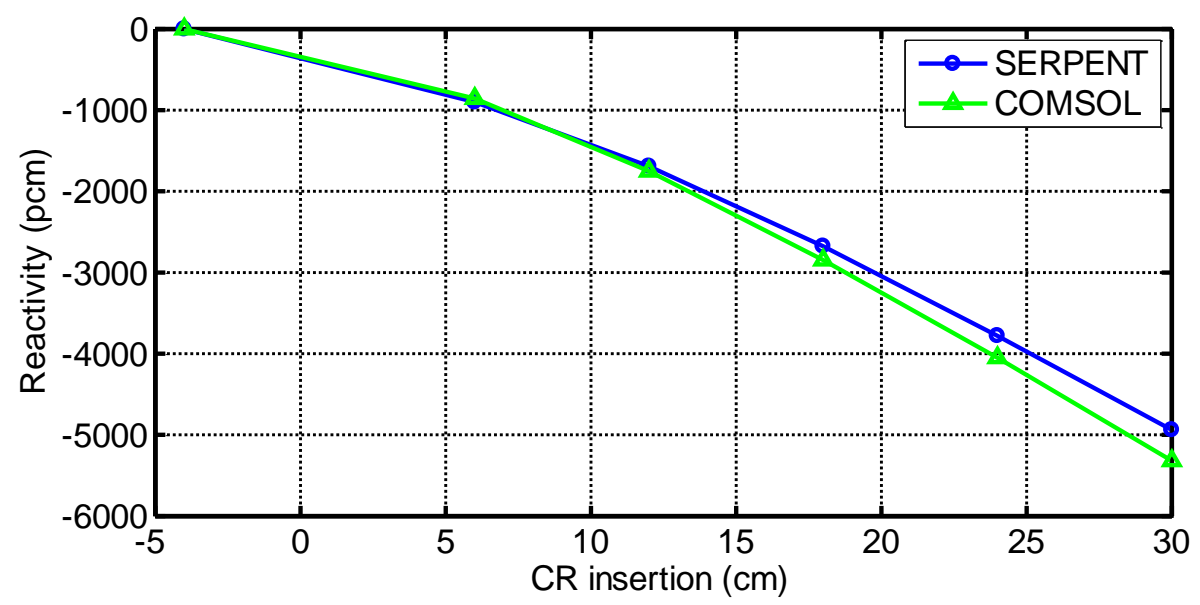

Figure 13. Reactivity curve of the control rods, comparison between SERPENT and COMSOL result (statistical errors are below $10 \mathrm{pcm}$ and are omitted from the plot). The overprediction of the CR worth in the COMSOL model can be explained by the use of the diffusion hypothesis.

Table 9. Comparison of SERPENT and COMSOL simulation results carried out to reproduce the CR insertion. The reactivity variation $(\mathrm{pcm})$ is taken as figure of merit to compare the results of the two modelling tools. The CR configurations present in this Table are used for the snapshots generation.

\begin{tabular}{ccccccc}
\hline $\begin{array}{c}\text { CR insertion } \\
\text { (relative height } \\
\text { in cm) }\end{array}$ & $\begin{array}{c}\text { SERPENT } \\
\text { (transport) }\end{array}$ & $\begin{array}{c}\text { COMSOL } \\
\text { (diffusion) }\end{array}$ & $\begin{array}{c}\text { Error } \\
(\%)\end{array}$ & $\begin{array}{c}\text { SERPENT } \\
\text { (transport) }\end{array}$ & $\begin{array}{c}\text { COMSOL } \\
\text { (diffusion) }\end{array}$ & $\begin{array}{c}\text { Error } \\
(\%)\end{array}$ \\
\hline-4 (extracted) & $1.07391 \pm 6 \cdot 10^{-5}$ & 1.07387 & 7.4 & 0 & 0 & -5.0 \\
6 & $1.06362 \pm 6 \cdot 10^{-5}$ & 1.06409 & 6.4 & $-901 \pm 7.5$ & -855.8 & -175 \\
12 & $1.05474 \pm 6 \cdot 10^{-5}$ & 1.05405 & 5.4 & $-1692 \pm 7.5$ & -1751.7 & 3.5 \\
18 & $1.0439 \pm 6 \cdot 10^{-5}$ & 1.04201 & 4.2 & $-2677 \pm 7.5$ & -2847.5 & 6.4 \\
24 & $1.03201 \pm 6 \cdot 10^{-5}$ & 1.02911 & 2.9 & $-3781 \pm 7.5$ & -4050 & 7.1 \\
30 & $1.01982 \pm 6 \cdot 10^{-5}$ & 1.01584 & 1.6 & $-4939 \pm 7.5$ & -5320.1 & 7.7 \\
\hline
\end{tabular}


The multiplication factor and the reactivity variation calculated with the spatial neutronics model in the different cases are reported in Table 10 and in Table 11, respectively. The CR configurations marked with an asterisk refers to CR settings that were not considered in the snapshots generation. This is meant to test the several methods in conditions far from the snapshots set and it gives indications about the reduced order model performance. Seven functions for the flux expansion are considered for the Cases A, B, C, D. The results give three indications. The first one is that the best performance is obtained in Case D (APOD method). The second one is that the Modal Method, both Cases A and B, are totally inadequate to reproduce the effect of the CR movement. This can be explained considering that the MM employs the spatial basis calculated in a unique reference solution. This means that it is suitable only if the flux is not strongly perturbed, which is not the case of the CR movement. Finally, the results are satisfactory also in conditions different from the snapshots set (marked in Table 10 and in Table 11 with an asterisk) indicating that the spatial neutronics model do not require a large number of full model simulations for achieving satisfactory results.

Table 10. CR insertion. Multiplication factor calculated by the spatial neutronics model using the different methods presented in the paper (seven spatial basis functions). The reference value is the reactivity calculated by the COMSOL model. Good results are obtained by the APOD method. The CR configurations marked with an asterisk refer to CR settings that were not consider in the snapshots generation

\begin{tabular}{|c|c|c|c|c|c|c|c|}
\hline \multirow{3}{*}{$\begin{array}{l}\text { CR insertion } \\
\text { (relative } \\
\text { height in } \mathrm{cm} \text { ) }\end{array}$} & \multicolumn{7}{|c|}{ Multiplication factor $(\mathrm{N}=7)$} \\
\hline & \multirow{2}{*}{ Case A } & \multirow{2}{*}{ Case B } & \multicolumn{2}{|c|}{ Case $\mathrm{C}$} & \multicolumn{2}{|c|}{ Case D } & \multirow{2}{*}{$\begin{array}{l}\text { COMSOL } \\
\text { (reference) }\end{array}$} \\
\hline & & & Value & Diff. $(\mathrm{pcm})$ & Value & Diff. (pcm) & \\
\hline-4 (extracted) & 1.075426 & 1.073484 & 1.07458 & -65.80 & 1.073864 & 0.82 & 1.073873 \\
\hline 6 & 1.052188 & 1.049962 & 1.06447 & -34.93 & 1.064055 & 3.63 & 1.064094 \\
\hline $9 *$ & 1.045540 & 1.043496 & 1.06033 & -32.30 & 1.059949 & 6.36 & 1.060013 \\
\hline 12 & 1.018336 & 1.015744 & 1.05485 & -76.10 & 1.053916 & 12.29 & 1.054045 \\
\hline $15^{*}$ & 1.005610 & 1.003080 & 1.04873 & -44.76 & 1.048675 & -38.89 & 1.048286 \\
\hline 18 & 0.976147 & 0.973072 & 1.04207 & -6.00 & 1.041890 & 11.52 & 1.042010 \\
\hline $21^{*}$ & 0.961381 & 0.958173 & 1.03579 & -33.89 & 1.035398 & 4.80 & 1.035446 \\
\hline 24 & 0.931826 & 0.928297 & 1.02980 & -66.11 & 1.029048 & 6.52 & 1.029115 \\
\hline $27^{*}$ & 0.916434 & 0.912620 & 1.02259 & -42.55 & 1.022053 & 10.77 & 1.022160 \\
\hline 30 & 0.889722 & 0.885887 & 1.01624 & -39.35 & 1.015817 & 1.93 & 1.015837 \\
\hline
\end{tabular}

Table 11. CR insertion. Reactivity variation $(\mathrm{pcm})$ calculated by the spatial neutronics model using the different methods presented in the paper (seven spatial basis functions). The reference value is the reactivity calculated by the COMSOL model. Good results are obtained by the APOD method. The CR configurations marked with an asterisk refer to $\mathrm{CR}$ settings that were not consider in the snapshots generation

\begin{tabular}{cccccccc}
\hline $\begin{array}{c}\text { CR insertion } \\
\text { (relative } \\
\text { height in cm) }\end{array}$ & Case A & Case B & \multicolumn{6}{c}{ Case C } & \multicolumn{2}{c}{ Case D } & COMSOL \\
\cline { 2 - 8 } & & & Value & Diff. (pcm) & Value & Diff. (pcm) & (reference) \\
\hline 6 & -2053.7 & -2086.9 & -884.2 & 28.4 & -858.4 & 2.64 & $\mathbf{- 8 5 5 . 8}$ \\
$9^{*}$ & -2657.9 & -2677.1 & -1250.2 & 32.62 & -1222.5 & 4.88 & $\mathbf{- 1 2 1 7 . 6 2}$ \\
12 & -5213.0 & -5295.4 & -1740.8 & -10.9 & -1762.6 & 10.90 & $\mathbf{- 1 7 5 1 . 7}$ \\
$15^{*}$ & -6455.8 & -6538.3 & -2293.5 & 20.56 & -2236.8 & -36.16 & $\mathbf{- 2 2 7 2 . 9 2}$ \\
18 & -9457.2 & -9612.7 & -2903.0 & 55.5 & -2857.8 & 10.29 & $\mathbf{- 2 8 4 7 . 5}$ \\
$21^{*}$ & -11030.7 & -11210.7 & -3485.5 & 29.67 & -3459.5 & 3.7 & $\mathbf{- 3 4 5 5 . 8 2}$ \\
24 & -14329.8 & -14569.6 & -4047.0 & -3.0 & -4055.6 & 5.57 & $\mathbf{- 4 0 4 9 . 9 9}$ \\
$27^{*}$ & -16132.2 & -16420 & -4731.7 & 20.56 & -4720.7 & 9.53 & $\mathbf{- 4 7 1 1 . 1 2}$ \\
30 & -19408.3 & -19726.6 & -5342.6 & 22.5 & -5321.2 & 1.14 & $\mathbf{- 5 3 2 0 . 1}$ \\
\hline
\end{tabular}


The evolution of the multiplication factor versus the number of the functions employed is described in Figure 14a (CR extracted) and in Figure 14b (CR height $18 \mathrm{~cm})$. Also in this case, the convergence to the reference result is reached by the APOD method (Case D) with less functions compared to the POD method (Case C). It is interesting to notice that in the APOD case the addition of a spatial basis function results in an improvement of the model performance (i.e., the error in the multiplication factor - compared to the COMSOL reference simulation - decreases monotonously with the number of the functions). This is not true for the Case C.

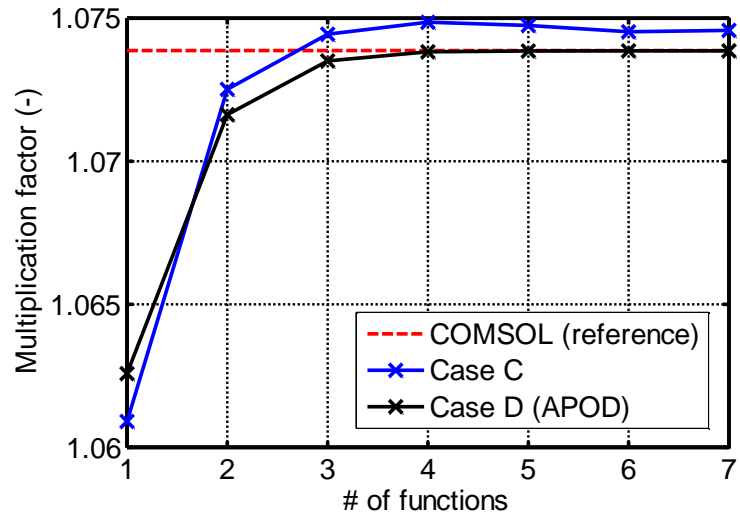

(a)

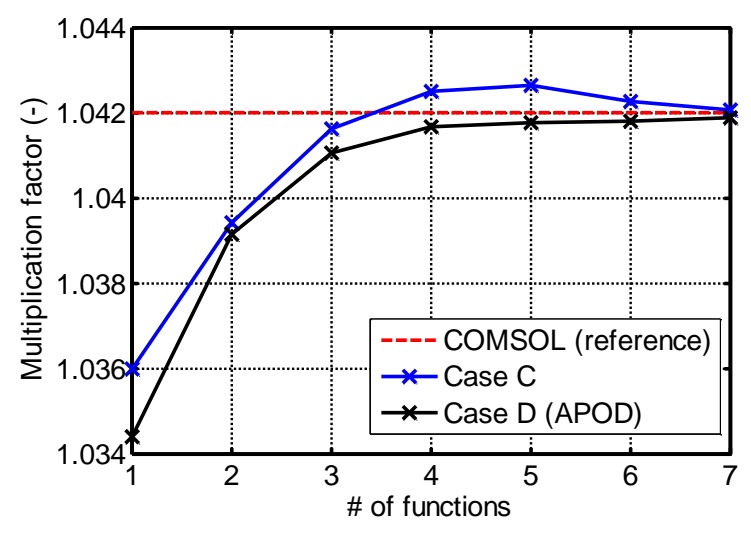

(b)

Figure 14. Evolution of the multiplication factor versus the number of functions employed in the model for the POD methods (Case C and APOD method) in two simulated configurations: (a) CR extracted and (b) CR height $18 \mathrm{~cm}$. Case $A$ and Case $B$ are not reported since they are not suitable to reproduce the reactivity in case of $C R$ movement. The APOD method shows a better performance with respect to the Case $C$.

\subsection{Discussion of results}

As far as the Modal Method is concerned, the results of the previous sections state that only in case of thermal reactivity effects, the approach gives acceptable results and in particular only if the adjoint eigenvectors are employed (Case B). If the eigenvectors of the flux are employed as test functions (Case A), the spatial neutronics model is not able at all to reproduce the reactivity. The Modal Method (both Cases A and B) is totally inappropriate for considering strong perturbation of the flux as happens during the control rod movements. Therefore, it is not the ideal candidate to be employed in the spatial neutronics model.

The problem of strong perturbation can be overcome employing the POD method for the selection of the spatial basis. The results show very good results for Case $\mathrm{C}$ (the test functions are the same of the POD modes) and Case D (the test functions take into account the adjoint flux). In particular, both for the thermal reactivity effects and the CR movement, the APOD method (Case D) shows better outcomes compared to the classic POD method for several reasons. Firstly, it reaches the best accuracy with less functions employed, meaning that the computational cost to run the model is reduced. Secondly, the APOD method is less affected by the dependence of the functions number on the convergence to the reference value, compared to the POD method. Thirdly, it obtains good results also in situations which are not included in the snapshots set. Finally, the best performance of the APOD method can be explained considering the role that the adjoint flux takes on in the perturbation theory. In this context, it is used as weighting function for the evaluation of the reactivity variation (Henry, 1975). Similarly, in the reduced order methods, the test functions are used to "evaluate" the 
residual introduced with the approximation of Equation (4) and constraining it to zero (Amsallem and Farhat, 2012).

As for the computational time, the spatial neutronics model allows a greater reduction of the computational effort, making this reduced order model suitable to be employed for real-time applications (Table 12).

Table 12. Computational time for a single eigenvalue calculation of the several approaches used in the paper. SERPENT and COMSOL models are run on workstations whereas the spatial neutronics model runs on a laptop.

\begin{tabular}{cccc}
\hline \multirow{2}{*}{ SERPENT } & \multirow{2}{*}{ COMSOL } & \multicolumn{2}{c}{ Spatial Neutronics Model } \\
\cline { 3 - 4 } & & $\mathrm{N}=5$ & $\mathrm{~N}=7$ \\
\hline $200 \mathrm{cpu}-\mathrm{h}$ & $5 \mathrm{cpu}-\mathrm{h}$ & $0.4 \mathrm{~ms}$ & $0.8 \mathrm{~ms}$ \\
\hline
\end{tabular}

\section{Conclusions}

In this work, different methods for the selection of the best spatial basis/test functions for a spatial neutronics model have been analysed. In order to obtain a neutronics modelling, which is both accurate and computationally efficient, the use of reduced order methods can be suitable. To this aim, the paradigm of the ROM is transferred into neutronics separating the spatial and time dependence of the neutron flux, which can be represented as a linear combination of spatial basis functions. The selection of the pair spatial basis/test functions is therefore crucial. In this paper, two different methods for the selection of the spatial basis (the Modal Method and the Proper Orthogonal Decomposition) and two options for the test functions (the same functions that constitute the spatial basis or their adjoint) have been tested. In particular, the Adjoint Proper Orthogonal Decomposition method has been developed aimed at combining the peculiarities of the Proper Orthogonal Decomposition and the use of the adjoint flux as test function in the neutronics framework.

A detailed model of the ALFRED reactor has been set up by means of the continuous energy Monte Carlo neutron transport code SERPENT with a heterogeneous description of the active zone. The average cross-sections for each assembly, calculated by means of the Monte Carlo model, have been used to solve the neutron diffusion PDEs. Starting from the spatial basis and the test functions calculated by means of the neutron diffusion equations, the MM has proved not to be suitable in case of CR movement even if it works for thermal feedback effects. As a major outcome of the simulation results, besides the capability of the model to take into account the spatial distribution of the reactivity, the Adjoint Proper Orthogonal Decomposition has turn out to be the best choice in reproducing the reactivity effects in terms of both accuracy and number of functions employed, the latter having an impact on the computational time. These results can be explained considering the role that the adjoint flux takes on in the perturbation theory as weighting function for the neutron importance.

In conclusion, a spatial neutronics model can be improved considering the APOD method for the selection of the spatial basis and the test functions. This reduced order model can be used whether both accuracy and computational efficiency are required at the same time. A possible application is the control context and the use of reduced order models in control-oriented simulations. The adopted description allows for the spatial heterogeneity of the system, in particular as far as the thermal reactivity feedbacks are concerned, providing a spatial representation of the neutron flux. On the other hand, it can be employed in control-oriented applications, being accurate in both the reactivity and transient representation (Lorenzi et al., 2015) without an excessive computational cost. This modelling improvement may allow adopting innovative control strategies, whose feasibility in the 
nuclear field cannot be adequately studied by means of the Point Kinetics approach. For instance, with a spatial neutronics model, an optimal control of the $\mathrm{CR}$ movement that minimizes the perturbation on the neutron flux can be assessed, since the model allows for the flux distortion due to the CR insertion. Another possible application is the sensitivity analysis and uncertainty quantification framework, where a fast-running model is useful to span the possible range of the input parameter and to propagate the uncertainty.

\section{Acknowledgements}

The author is grateful to A. Cammi and L. Luzzi (Politecnico di Milano, Italy) for the fruitful conversation and suggestions about the paper contents. 


\section{List of symbols}

\section{Latin Symbols}

A coefficient used in Eq. (11), $\mathrm{cm}^{-1}$

$C_{j} \quad$ concentration of the $\mathrm{j}^{\text {th }}$ precursor group, $\mathrm{cm}^{-3}$

$D_{g} \quad$ neutron diffusion coefficient of the $\mathrm{g}^{\text {th }}$ energy group, $\mathrm{cm}$

E energy, $\mathrm{MeV}$

$E_{g} \quad$ energy group threshold, eV

$\mathrm{G}$ number of energy group, -

$\mathrm{h}_{\mathrm{CR}} \quad$ height of control rods, $\mathrm{m}$

$N$ number of employed functions in the spatial basis, -

$N_{S} \quad$ number of snapshots in the POD method, -

$N_{p} \quad$ number of employed POD for the spatial basis, -

$\boldsymbol{n}$ surface normal unit vector, -

$n_{i}^{g} \quad$ time-dependent coefficient of the $\mathrm{i}^{\text {th }}$ spatial function of the neutron flux of the $\mathrm{g}^{\text {th }}$ energy group, -

$\boldsymbol{r} \quad$ spatial coordinate, $\mathrm{cm}$

$\mathrm{S} \quad$ surface of the spatial domain, $\mathrm{cm}^{2}$

$T$ temperature, $\mathrm{K}$

$t \quad$ time, $\mathrm{s}$

$v_{g} \quad$ neutron speed of the $\mathrm{g}^{\text {th }}$ energy group, $\mathrm{cm} \mathrm{s}^{-1}$

\section{Greek Symbols}

$\beta \quad$ total delayed neutron fraction, $\mathrm{pcm}$

$\beta_{j} \quad$ delayed neutron fraction of the $\mathrm{j}^{\text {th }}$ precursor group, $\mathrm{pcm}$

$\gamma \quad$ albedo coefficient used in Eq. (12), -

$\lambda_{j} \quad$ decay constant of the $\mathrm{j}^{\text {th }}$ precursor group, $\mathrm{s}^{-1}$

$\lambda_{i}^{*} \quad \mathrm{i}^{\text {th }}$ eigenvalue, -

$v \quad$ average number of neutrons emitted per fission event, -

$\xi_{i}^{g} \quad \mathrm{i}^{\text {th }}$ test function of the $\mathrm{g}^{\text {th }}$ energy group, -

$\rho$ reactivity, $\mathrm{pcm}$

$\Sigma \quad$ generic macroscopic cross-section, $\mathrm{cm}^{-1}$

$\Sigma_{a}^{g} \quad$ macroscopic absorption cross-section of the $\mathrm{g}^{\text {th }}$ energy group, $\mathrm{cm}^{-1}$

$\Sigma_{f}^{g} \quad$ macroscopic fission cross-section of the $\mathrm{g}^{\text {th }}$ energy group, $\mathrm{cm}^{-1}$

$\Sigma_{s}^{g \rightarrow} \quad$ macroscopic cross-section including scattering out of the energy group $\mathrm{g}, \mathrm{cm}^{-1}$

$\sum_{s}^{g \rightarrow g^{\prime}}$ macroscopic group transfer cross-section from energy group $\mathrm{g}$ to $\mathrm{g}^{\prime}, \mathrm{cm}^{-1}$

$\phi_{g} \quad$ neutron flux of the $\mathrm{g}^{\text {th }}$ energy group, $\mathrm{cm}^{-2} \mathrm{~s}^{-1}$

$\chi_{d}^{g} \quad$ fraction of delayed neutrons generated in the $\mathrm{g}^{\text {th }}$ energy group, -

$\chi_{p}^{g} \quad$ fraction of prompt neutrons generated in the $\mathrm{g}^{\text {th }}$ energy group, -

$\chi_{t}^{g} \quad$ fraction of total neutrons generated in the $\mathrm{g}^{\text {th }}$ energy group, -

$\psi_{i}^{g} \quad \mathrm{i}^{\text {th }}$ spatial eigenfunction of the neutron flux of the $\mathrm{g}^{\text {th }}$ energy group, $\mathrm{cm}^{-2} \mathrm{~s}^{-1}$

$\Omega \quad$ spatial domain, $\mathrm{cm}^{3}$

\section{Subscripts}

0 reference value

a axial

f fuel

g energy group number 
gz generic zone

$\mathrm{r}$ radial 


\section{References}

Abdel-Khalik, H.S., Bang, Y., Wang, C., 2013. Overview of hybrid subspace methods for uncertainty quantification, sensitivity analysis. Ann. Nucl. Energy 52, 28-46. doi:10.1016/j.anucene.2012.07.020

Alemberti, A., De Bruyn, D., Grasso, G., Mansani, L., Mattioli, D., Roelofs, F., 2013a. The European Lead Fast Reactor strategy and the Roadmap for the Demonstrator ALFRED, in: Proceedings of the IAEA International Conference on Fast Reactors and Related Fuel Cycles: Safe Technologies and Sustainable Scenarios (FR13), France, March 4-7, 2013.

Alemberti, A., Frogheri, M., Mansani, L., 2013b. THE LEAD FAST REACTOR: DEMONSTRATOR (ALFRED) AND ELFR DESIGN, in: Proceedings of the IAEA International Conference on Fast Reactors and Related Fuel Cycles: Safe Technologies and Sustainable Scenarios (FR13), France, March 4-7, 2013.

Alemberti, A., Smirnov, V., Smith, C.F., Takahashi, M., 2014. Overview of lead-cooled fast reactor activities. Prog. Nucl. Energy 77, 300-307. doi:10.1016/j.pnucene.2013.11.011

Amsallem, D., Farhat, C., 2012. Stabilization of projection-based reduced-order models. Int. J. Numer. Methods Eng. 91, 358-377. doi:10.1002/nme.4274

Aufiero, M., Fratoni, M., 2017. Stabilization and convergence acceleration in coupled Monte Carlo - CFD calculations : the Newton method via Monte Carlo Perturbation Theory, in: M\&C 2017 - International Conference on Mathematics \& Computational Methods Applied to Nuclear Science \& Engineering, Jeju, Korea, April 16-20, 2017.

Aufiero, M., Martin, M., Fratoni, M., 2016. XGPT: Extending Monte Carlo Generalized Perturbation Theory capabilities to continuous-energy sensitivity functions. Ann. Nucl. Energy 96, 295-306. doi:10.1016/j.anucene.2016.06.012

Bang, Y., Abdel-Khalik, H.S., Hite, J.M., 2012a. Hybrid reduced order modeling applied to nonlinear models. Int. J. Numer. Methods Eng. 91, 929-949. doi:10.1002/nme.4298

Bang, Y., Abdel-Khalik, H.S., Jessee, M.A., Mertyurek, U., 2015. Hybrid reduced order modeling for assembly calculations. Nucl. Eng. Des. 295, 661-666. doi:10.1016/j.nucengdes.2015.07.020

Bang, Y., Wang, C., Abdel-Khalik, H.S., 2012b. State-Based Adjoint Method for Reduced Order Modeling. Transp. Theory Stat. Phys. 41, 101-132. doi:10.1080/00411450.2012.672359

Berkooz, G., Holmes, P., Lumley, J.L., 1993. The proper orthogonal decomposition in the analysis of turbulent flows. Annu. Rev. Fluid Mech. 25, 539-575.

Buchan, A.G., Calloo, A.A., Goffin, M.G., Dargaville, S., Fang, F., Pain, C.C., Navon, I.M., 2015. A POD reduced order model for resolving angular direction in neutron/photon transport problems. J. Comput. Phys. 296, 138-157. doi:10.1016/j.jcp.2015.04.043

Buchan, A.G., Pain, C.C., Fang, F., Navon, I.M., 2013. A POD reduced-order model for eigenvalue problems with application to reactor physics. Int. J. Numer. Methods Eng. 95, 1011-1032. doi:10.1002/nme.4533

Chinesta, F., Huerta, A., Rozza, G., Wilcox, K., 2016. Model order reduction. Encycl. Comput. Mech.

Cinotti, L., Locatelli, G., Aït Abderrahim, H., Monti, S., Benamati, G., Tucek, K., Struwe, D., Orden, A., Corsini, G., Le Carpentier, D., 2008. The ELSY Project, in: Proceedings of the International Conference on the Physics of Reactors Nuclear Power: A Sustainable Resource (PHYSOR 08), Interlaken, Switzerland, September 14-19.

COMSOL, 2011. Comsol Multiphysics 4.2a, User's Guide. 
Duderstadt, J.J., Hamilton, L.J., 1976. Nuclear Reactor Analysis. John Wley and Sons, New York.

Gong, H., Argaud, J.P., Bouriquet, B., Maday, Y., 2016. The Empirical Interpolation Method Applied To the Neutron Diffusion Equations With Parameter Dependence, in: Proceedings of Physics of Reactors Conference: Unifying Theory and Experiments in the 21st Century (PHYSOR 2016), Sun Valley, Idaho, USA, May 1-5. pp. 54-63.

Grasso, G., Petrovich, C., Mattioli, D., Artioli, C., Sciora, P., Gugiu, D., Bandini, G., Bubelis, E., Mikityuk, K., 2014. The core design of ALFRED, a demonstrator for the European lead-cooled reactors. Nucl. Eng. Des. 278, 287-301. doi:10.1016/j.nucengdes.2014.07.032

Gunzburger, M.D., 2002. Perspectives in Flow Control and Optimization. Society for Industrial and Applied Mathematics. doi:10.1137/1.9780898718720

Henry, A.F., 1975. Nuclear reactor analysis. MIT Press.

Hesthaven, J.S., Rozza, G., Stamm, B., 2016. Certified Reduced Basis Methods for Parametrized Partial Differential Equations, SpringerBriefs in Mathematics. Springer International Publishing, Cham. doi:10.1007/978-3-319-22470-1

Holmes, P., Lumley, J.L., Berkooz, G., 1996. Turbulence, Coherent Structures, Dynamical Systems and Symmetry. Cambridge University Press, Cambridge, UK.

Koning, A., 2006. The JEFF-3.1 Nuclear Data Library. JEFF-3.1 Nucl. Data Libr.

Leppänen, J., Pusa, M., Viitanen, T., Valtavirta, V., Kaltiaisenaho, T., 2015. The Serpent Monte Carlo code: Status, development and applications in 2013. doi:10.1016/j.anucene.2014.08.024

Lorenzi, S., Cammi, A., Luzzi, L., Ponciroli, R., 2015. A Control-Oriented Modeling Approach to Spatial Neutronics Simulation of a Lead-Cooled Fast Reactor. J. Nucl. Eng. Radiat. Sci. doi:10.1115/1.4029791

Lorenzi, S., Cammi, A., Luzzi, L., Rozza, G., 2017. A reduced order model for investigating the dynamics of the Gen-IV LFR coolant pool. Appl. Math. Model. 46, 263-284. doi:10.1016/j.apm.2017.01.066

Lorenzi, S., Cammi, A., Luzzi, L., Rozza, G., 2016. POD-Galerkin method for finite volume approximation of Navier-Stokes and RANS equations. Comput. Methods Appl. Mech. Eng. 311, 151-179. doi:10.1016/j.cma.2016.08.006

Manzoni, A., Quarteroni, A., Rozza, G., 2012. Computational Reduction for Parametrized PDEs: Strategies and Applications. Milan J. Math. 80, 283-309. doi:10.1007/s00032-012-0182-y

MATLAB, 2016. The MathWorks, Inc.

Quarteroni, A., Manzoni, A., Negri, F., 2016. Reduced Basis Methods for Partial Differential Equations, UNITEXT. Springer International Publishing, Cham. doi:10.1007/978-3-319-154312

Quarteroni, A., Rozza, G., Manzoni, A., 2011. Certified reduced basis approximation for parametrized partial differential equations and applications. J. Math. Ind. 1, 1-49. doi:10.1186/2190-5983-1-3

Rozza, G., Huynh, D.B.P., Patera, A.T., 2008. Reduced basis approximation and a posteriori error estimation for affinely parametrized elliptic coercive partial differential equations: Application to transport and continuum mechanics. Arch. Comput. Methods Eng. 15, 229-275. doi:10.1007/s11831-008-9019-9

Sartori, A., Baroli, D., Cammi, A., Chiesa, D., Luzzi, L., Ponciroli, R., Previtali, E., Ricotti, M.E., Rozza, G., Sisti, M., 2014. Comparison of a Modal Method and a Proper Orthogonal Decomposition approach for multi-group time-dependent reactor spatial kinetics. Ann. Nucl. 
Energy 71. doi:10.1016/j.anucene.2014.03.043

Sirovich, L., 1987. Turbulence and the dynamics of coherent structures. I - Coherent structures. Quart. Appl. Math. 45, 561-571.

Sorensen, D.C., 2002. Numerical methods for large eigenvalue problems. Acta Numer. 11, 519-584. doi:10.1017/S0962492902000089

Stacey, W.M., 1969. Space-time nuclear reactor kinetics. Academic Press, New York.

Volkwein, S., 1999. Proper Orthogonal Decomposition and Singular Value Decomposition. 